\title{
Chemistry and Star Formation in the Host Galaxies of Type la Supernovae
}

\section{Citation}

Gallagher, Joseph S., Peter M. Garnavich, Perry Berlind, Peter Challis, Saurabh Jha, and Robert P. Kirshner. 2005. "Chemistry and Star Formation in the Host Galaxies of Type la Supernovae." The Astrophysical Journal 634 (1): 210-26. https://doi.org/10.1086/491664.

\section{Permanent link}

http://nrs.harvard.edu/urn-3:HUL.InstRepos:41399918

\section{Terms of Use}

This article was downloaded from Harvard University's DASH repository, and is made available under the terms and conditions applicable to Other Posted Material, as set forth at http:// nrs.harvard.edu/urn-3:HUL.InstRepos:dash.current.terms-of-use\#LAA

\section{Share Your Story}

The Harvard community has made this article openly available.

Please share how this access benefits you. Submit a story.

Accessibility 
The Astrophysical Journal, 634:210-226, 2005 November 20

(C) 2005. The American Astronomical Society. All rights reserved. Printed in U.S.A.

\title{
CHEMISTRY AND STAR FORMATION IN THE HOST GALAXIES OF TYPE Ia SUPERNOVAE
}

\author{
Joseph S. Gallagher and Peter M. Garnavich \\ Department of Physics, University of Notre Dame, 225 Nieuwland Science Hall, Notre Dame, IN 46556-5670 \\ Perry Berlind \\ F. L. Whipple Observatory, 670 Mount Hopkins Road, P.O. Box 97, Amado, AZ 85645 \\ Peter Challis \\ Harvard-Smithsonian Center for Astrophysics, 60 Garden Street, Cambridge, MA 02138 \\ SAURABH JHA \\ Department of Astronomy, 601 Campbell Hall, University of California, Berkeley, CA 94720-3411 \\ AND \\ ROBERT P. KIRSHNER \\ Harvard-Smithsonian Center for Astrophysics, 60 Garden Street, Cambridge, MA 02138 \\ Received 2005 March 4; accepted 2005 July 22
}

\begin{abstract}
We study the effect of environment on the properties of Type Ia supernovae by analyzing the integrated spectra of 57 local Type Ia supernova host galaxies. We deduce from the spectra the metallicity, current star formation rate, and star formation history of the host and compare these to the supernova decline rates. Additionally, we compare the host properties to the difference between the derived supernova distance and the distance determined from the best-fit Hubble law. From this we investigate possible uncorrected systematic effects inherent in the calibration of Type Ia supernova luminosities using light-curve fitting techniques. Our results indicate a statistically insignificant correlation in the direction of higher metallicity spiral galaxies hosting fainter Type Ia supernovae. However, we present qualitative evidence suggesting that progenitor age is more likely to be the source of variability in supernova peak luminosities than is metallicity. We do not find a correlation between the supernova decline rate and host galaxy absolute $B$ magnitude, nor do we find evidence of a significant relationship between decline rate and current host galaxy star formation rate. A tenuous correlation is observed between the supernova Hubble residuals and host galaxy metallicities. Further host galaxy observations will be needed to refine the significance of this result. Finally, we characterize the environmental property distributions for Type Ia supernova host galaxies through a comparison with two larger, more general galaxy distributions using Kolmogorov-Smirnov tests. The results show the host galaxy metallicity distribution to be similar to the metallicity distributions of the galaxies of the NFGS and SDSS. Significant differences are observed between the SN Ia distributions of absolute $B$ magnitude and star formation histories and the corresponding distributions of galaxies in the NFGS and SDSS. Among these is an abrupt upper limit observed in the distribution of star formation histories of the host galaxy sample, suggesting a Type Ia supernovae characteristic delay time lower limit of approximately 2.0 Gyr. Other distribution discrepancies are investigated and the effects on the supernova properties are discussed.
\end{abstract}

Subject headings: distance scale - supernovae: general

\section{INTRODUCTION}

Among the powerful tools that have come into prominence in the last decade in the field of cosmology, few have been as important in advancing the subject as Type Ia supernovae ( $\mathrm{SNe} \mathrm{Ia}$ ). $\mathrm{SNe}$ Ia show variations in their peak luminosities and colors that correlate well with their light-curve decay times (Phillips 1993; Hamuy et al. 1996b; Reiss et al. 1996; Perlmutter et al. 1999), making SNe Ia the best distance indicators. Application of this empirical relationship to the low-z sample of galaxies enabled cosmologists to refine the measurement of the Hubble constant with great precision (Jha et al. 1999; Freedman et al. 2001).

Realizing the potential for SNe Ia to act as accurate cosmological probes, researchers applied the technique to the high- $z$ sample of galaxies (Perlmutter et al. 1997; Garnavich et al. 1998; Schmidt et al. 1998). This research has yielded evidence suggesting that our universe is in a state of accelerating expansion, implying a form of dark energy whose nature we do not yet understand (Riess et al. 1998; Perlmutter et al. 1999). The goal of the ESSENCE project ${ }^{1}$ is to improve our understanding of this negative pressure by placing tight constraints on the cosmic equation of state through a study of $\sim 200 \mathrm{SNe}$ Ia at intermediate redshifts. The result will be a detailed map of the history of cosmic expansion with less than $2 \%$ distance error in six redshift bins and the ability to constrain the equation of state to $10 \%$. Moreover, future studies will attempt to differentiate between a vacuum energy and other more exotic sources for the acceleration (Wang \& Garnavich 2001; Miknaitis et al. 2005, in preparation), which will push the limits of the SNe Ia reliability. Clearly, it is of paramount importance to understand any systematic uncertainties in the calibration of $\mathrm{SNe}$ Ia that could bias these cosmological measurements.

Type Ia supernovae are identified by the presence of singly ionized silicon, magnesium, sulfur, calcium, and the conspicuous absence of hydrogen in their spectra. Early statistical studies

${ }^{1}$ See http://www.ctio.noao.edu/ wsne/index.html. 
of Type I supernovae and their host galaxies showed that these supernova events, like core-collapse $\mathrm{SNe}$, are associated with young stellar populations (Oemler \& Tinsley 1979; Caldwell \& Oemler 1981). However, unlike their core-collapse counterparts, Type Ia supernovae are readily observed far from the spiral arms in spiral galaxies and in early-type galaxies with low star formation rates (SFRs). These observations require a delay between formation and explosion that is long enough to allow for proper diffusion away from the spiral arms (McMillan \& Ciardullo 1996) and imply a lower mass for the SNe Ia progenitors. Specifically, $\mathrm{SNe}$ Ia are thought to be triggered by thermonuclear ignition in the core of a CO white dwarf (WD) near the Chandrasekhar mass limit $\left(1.4 M_{\odot}\right)$. Two models predict how the WD attains the mass necessary to initiate explosive burning. The first model is the single degenerate model (Whelan \& Iben 1973; Nomoto 1982), which describes a binary system in which a WD accretes matter from a main sequence or red giant binary companion. The second model, the double degenerate model (Webbink 1984; Iben \& Tutukov 1984), describes the coalescing of two binary WDs whose combined mass exceeds the Chandrasekhar mass limit. Once the mass limit is reached, carbon ignites, resulting in the outward propagation of a burning front from the WD core.

Detonation occurs if the burning front travels outward faster than the local sound speed, but such an explosion would convert most of the star to nickel and would leave too few intermediatemass elements compared to the observed spectra. A deflagration results when the flame front traverses the star subsonically, but this tends to produce too little kinetic energy to account for the observed velocities. A combination of these two scenarios, known as the delayed detonation (DD) model (Khokhlov 1991; Yamaoka et al. 1992; Woosley \& Weaver 1994) appears to best fit the observations. The DD model assumes a flame front propagation velocity that begins as deflagration and subsequently transitions into detonation at a specific transition density. Although the DD model has been able to match many of the features observed in $\mathrm{SNe}$ Ia, there remain many open questions. For example, what triggers the transition to a detonation and how does the WD build mass to reach the Chandrasekhar limit?

These uncertainties reinforce the need to investigate systematic effects that can influence the luminosity-decline rate relation. One important effect concerns the possible evolutionary changes undergone by the stellar populations producing the supernova progenitors. For example, systematic differences between the high- $z$ host stellar populations and the local host stellar populations could contribute to an inherent difference between the peak luminosities of low- $z$ events and those of the high- $z$ events. Fortunately, the local sample of galaxies provides such a wide range of host stellar environments that a study of these local environments can provide insight into environmental parameters that may correlate with redshift.

Theoretical models have shown that parameters such as progenitor mass and metallicity can have an effect on the luminosity and light-curve shape of the resultant supernova by influencing the relative $\mathrm{CNO}$ abundances in the white dwarf. For the DD model, massive progenitors produce faint Type Ia supernovae because of a low carbon fraction in the core (Höflich et al. 1998; Umeda et al. 1999). The carbon fraction is also lowered as the progenitor metallicity is increased, resulting in less energetic explosions. For the range of masses expected for $\mathrm{CO}$ white dwarfs, lowering the carbon fraction is expected to affect the peak brightness of Type Ia events by about $20 \%$. The range in peak brightness due to progenitor metallicity variations is expected to be small unless the metal abundance is significantly higher than solar (Timmes et al. 2003, hereafter TBT03).
For predictions such as these to be tested observationally, it would be necessary to analyze a large sample of Type Ia supernova host stellar populations covering a wide range of ages and metallicities against the parameters of the supernovae they produce. Unfortunately, it is difficult to isolate and observe the specific stellar populations harboring the progenitor systems. Moreover, a long delay between formation and explosion would blur the correlation between a SN characteristics and its present local environment. Consequently, the majority of observational research in this topic has centered on the study of the integrated light from the $\mathrm{SN}$ Ia host galaxies. An analysis of the integrated light has the added advantage of allowing for future comparisons with high- $z$ host galaxies whose small angular size restrict the observations to integrated spectra.

We characterize the SN environments through the spectroscopic study of 57 Type Ia supernova host galaxies. We have two goals for this study. The first is to take a direct look at the possible systematic effects that the host galaxy environment has on the SN Ia properties through an analysis of the interdependencies between host galaxy and SN Ia parameters. Second, we take an indirect look at these systematics by comparing our SN host sample with two larger, more general samples of galaxies - the galaxies of the Near Field Galaxy Survey (NFGS) and those of the Sloan Digital Sky Survey (SDSS). In $\S 2$ we introduce the host galaxy sample, detail the observing strategy, and present the data reduction process. In $\S 3$ the spectroscopic results are presented, and the theoretical predictions are discussed in light of the results. Finally, we summarize our conclusions in $\S 3.4$.

\section{OBSERVATIONS AND DATA REDUCTION}

\subsection{Observations}

The host galaxy spectra reported here were obtained with the FAST spectrograph (Fabricant et al. 1998) at the F. L. Whipple Observatory's $1.5 \mathrm{~m}$ Tillinghast telescope atop Mount Hopkins in Arizona. The data were taken during 13 nights from 1999 May through 2000 September. The seeing ranged from $1^{\prime \prime}$ to $2^{\prime \prime}$ throughout the survey. The FAST spectrograph, with a 300 line $\mathrm{mm}^{-1}$ reflection grating, allowed for $4000 \AA$ coverage and a FWHM resolution of $\sim 6 \AA$. The slit was $3^{\prime \prime}$ wide and had an unvignetted length of $3^{\prime}$.

The slit was aligned along each host galaxy's major axis to maximize the galactic light sampled. The position angles (P.A.'s) for each major axis were determined using the Digital Sky Survey (DSS) plates. The slit was offset to a distance matching the visible limit of the galaxy's minor axis on the DSS, and the slit was scanned repeatedly across the galaxy during an exposure. Exposure times ranged from 300 to $1200 \mathrm{~s}$, depending on the brightness of the target. Seven of the target galaxies (NGC 2841, NGC 3368, NGC 3627, NGC 4526, NGC 4527, NGC 4536, and NGC 5005) had major axes that subtended angles larger than $3^{\prime}$. In these cases, we oriented the slit along the galaxy's minor axis and scanned along its major axis. It should be noted that light losses due to atmospheric refraction are expected to be minimal given our use of a relatively wide $3^{\prime \prime}$ slit and the fact that this slit was scanned across the entire visual extent of our galaxies, an extent that typically measured many times the width of our slit.

At the beginning and the end of each night's run, both $12 \mathrm{~s}$ flat exposures and bias exposures were taken. Sky flats were taken to normalize the sensitivity along the slit. Flux standard star exposures were obtained twice per night with the slit oriented along the star's parallactic angle (Filippenko 1982). The standards were taken from the list given in Massey et al. (1988). Preceding the observation of every object galaxy, we obtained a comparison 
spectrum of a He-Ne-Ar lamp for reference in the wavelength calibration. For every object galaxy, save a few that will be addressed later, three images were taken with identical exposure times. Table 1 details our galaxy sample and the relevant observational parameters pertaining to each. Columns (1) and (2) give the common name of the target galaxy and the name of the supernova that it hosted, respectively. Column (3) shows the P.A. of the slit for each object, while the scan width is recorded in column (4). Column (5) gives the angular width of the extracted aperture for each host galaxy chosen to enclose all of the galactic light.

\subsection{Data Reduction}

The data reduction performed during this study was conducted following the standard techniques in the IRAF ${ }^{2}$ environment. The data were both dark- and bias-subtracted. Each galaxy spectrum was flat-fielded to correct for pixel-to-pixel variability in the CCD detector. Several pixels in each image were bad due to flaws on the CCD chip and had to be removed by interpolation. This was accomplished using the fixpix routine in IRAF. The acquisition of three identical spectra for each target galaxy allowed us to remove the majority of our cosmic rays by combining our images using the median parameter in the IRAF routine imcombine. Any further anomalous pixels were removed individually using imedit.

There were two conditions under which we were unable to remove cosmic rays in this fashion. First, in a few cases time constraints or poor atmospheric conditions prevented the acquisition of three spectra for the given target galaxy. In these situations, the cosmic rays were removed individually from those spectra we did obtain, and the images were averaged using imcombine. Second, to determine whether those objects with three images could be combined successfully using the median parameter in imcombine, it was necessary to ensure both that the spatial axes of each spectrum were aligned and that they each had comparable background levels. Both of these tasks were accomplished using the implot routine to plot the average of several cuts along each image's respective spatial axis. If the spatial axes were misaligned, they were shifted using the imshift routine in IRAF. Occasionally, short-term atmospheric variability resulted in evident variations observed in the continuum levels of the three-image set. If it was discernible which image(s) was bad, then that image(s) was removed and cosmic-ray removal proceeded as detailed above on the remaining spectra. On the other hand, if the anomalous image(s) was not evident, then the cosmic rays were removed individually from each image, aperture extraction was performed, and the extracted apertures were averaged using imcombine.

The next step was to extract a one-dimensional spectrum from each combined image using the apall routine in IRAF. The apertures were fitted interactively in IRAF and chosen to span a region on the spatial axis that extended slightly into the sky portion of the image on either side of the galaxy spectrum. In this way we ensured the inclusion of nearly $100 \%$ of the galactic light. However, attempts were made to avoid the inclusion of foreground stars in the aperture. The sky levels and trace were defined interactively using apall with a linear fit for the former and a third-order cubic spline for the latter. Wavelength and flux calibration proceeded using the standard techniques in IRAF.

\footnotetext{
2 IRAF is the Image Reduction and Analysis Facility, a general purpose software system for the reduction and analysis of astronomical data. IRAF is written and supported by the IRAF programming group at the National Optical Astronomy Observatory (NOAO). NOAO is operated by the Association of Universities for Research in Astronomy, Inc., under cooperative agreement with the National Science Foundation.
}

TABLE 1

The Host Galaxy Sample

\begin{tabular}{|c|c|c|c|c|}
\hline $\begin{array}{l}\text { Galaxy } \\
\text { (1) }\end{array}$ & $\begin{array}{l}\text { SN } \\
\text { (2) }\end{array}$ & $\begin{array}{l}\text { P.A. } \\
\text { (3) }\end{array}$ & $\begin{array}{l}\text { Scan } \\
\text { Width } \\
(4)\end{array}$ & $\begin{array}{l}\text { Aperture } \\
\text { Width } \\
\text { (5) }\end{array}$ \\
\hline NGC 4536............................... & 1981B & 30 & $120^{\prime \prime}$ & 51 \\
\hline 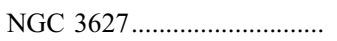 & 1989B & 90 & $3^{\prime}$ & 71 \\
\hline 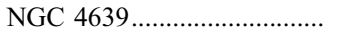 & $1990 \mathrm{~N}$ & -10 & $1^{\prime}$ & 51 \\
\hline CGCG $111016 \ldots \ldots \ldots \ldots \ldots$ & 19900 & -67 & $15^{\prime \prime}$ & 23 \\
\hline 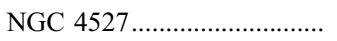 & $1991 \mathrm{~T}$ & -25 & $120^{\prime \prime}$ & 46 \\
\hline IC $4232 \ldots$ & $1991 U$ & 5 & $20^{\prime \prime}$ & 31 \\
\hline NGC $4374 \ldots$ & $1991 \mathrm{bg}$ & -50 & $3^{\prime}$ & 38 \\
\hline Anon................. & $1992 J$ & 85 & $15^{\prime \prime}$ & 26 \\
\hline IC 3690 . & $1992 \mathrm{P}$ & -5 & $15^{\prime \prime}$ & 18 \\
\hline 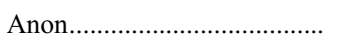 & 1992ag & 100 & $15^{\prime \prime}$ & 29 \\
\hline 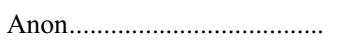 & $1992 \mathrm{bp}$ & 40 & $15^{\prime \prime}$ & 17 \\
\hline CGCG 307 023........................ & $1993 \mathrm{ac}$ & 150 & $15^{\prime \prime}$ & 18 \\
\hline 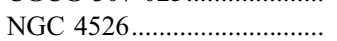 & 1994D & 20 & $90^{\prime \prime}$ & 46 \\
\hline NGC $4493 \ldots \ldots . . .$. & $1994 \mathrm{M}$ & -20 & $20^{\prime \prime}$ & 34 \\
\hline 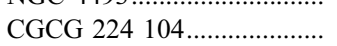 & 1994Q & 70 & $10^{\prime \prime}$ & 14 \\
\hline 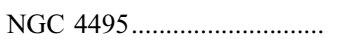 & $1994 \mathrm{~S}$ & -45 & $20^{\prime \prime}$ & 46 \\
\hline NGC 3370................................ & 1994ae & -35 & $1^{\prime}$ & 23 \\
\hline 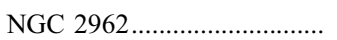 & 1995D & -5 & $1^{\prime}$ & 46 \\
\hline 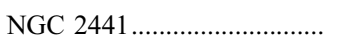 & $1995 \mathrm{E}$ & -20 & $1^{\prime}$ & 51 \\
\hline IC 1844 & $1995 \mathrm{ak}$ & 100 & $15^{\prime \prime}$ & 36 \\
\hline NGC $3021 \ldots \ldots$. & $1995 \mathrm{al}$ & 100 & $30^{\prime \prime}$ & 46 \\
\hline 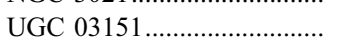 & $1995 \mathrm{bd}$ & 100 & $15^{\prime \prime}$ & 51 \\
\hline 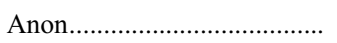 & $1996 \mathrm{C}$ & 0 & $15^{\prime \prime}$ & 23 \\
\hline 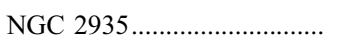 & $1996 Z$ & -30 & $2^{\prime}$ & 49 \\
\hline Anon & $1996 \mathrm{ab}$ & -30 & $0^{\prime \prime}$ & 15 \\
\hline 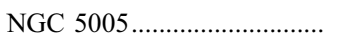 & 1996ai & -25 & $2^{\prime}$ & 51 \\
\hline 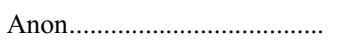 & 1996bl & 80 & $15^{\prime \prime}$ & 17 \\
\hline 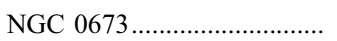 & 1996bo & 0 & $45^{\prime \prime}$ & 54 \\
\hline 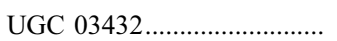 & $1996 \mathrm{bv}$ & 130 & $15^{\prime \prime}$ & 46 \\
\hline NGC 2258............................... & $1997 \mathrm{E}$ & 150 & $15^{\prime \prime}$ & 29 \\
\hline NGC $4680 \ldots$. & 1997bp & 30 & $20^{\prime \prime}$ & 40 \\
\hline 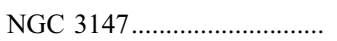 & $1997 \mathrm{bq}$ & 0 & $90^{\prime \prime}$ & 68 \\
\hline Anon & $1997 \mathrm{br}$ & -20 & $20^{\prime \prime}$ & 63 \\
\hline 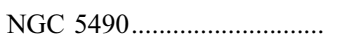 & $1997 \mathrm{cn}$ & 10 & $20^{\prime \prime}$ & 34 \\
\hline 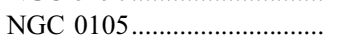 & $1997 \mathrm{cw}$ & 0 & $30^{\prime \prime}$ & 46 \\
\hline 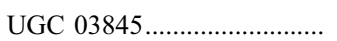 & 1997do & -10 & $30^{\prime \prime}$ & 46 \\
\hline 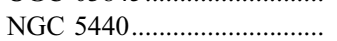 & $1998 \mathrm{D}$ & 50 & $30^{\prime \prime}$ & 23 \\
\hline NGC $6627 \ldots$. & $1998 \mathrm{~V}$ & 80 & $15^{\prime \prime}$ & 34 \\
\hline 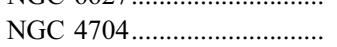 & $1998 \mathrm{ab}$ & 45 & $30^{\prime \prime}$ & 29 \\
\hline 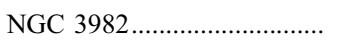 & $1998 \mathrm{aq}$ & 90 & $1^{\prime}$ & 57 \\
\hline 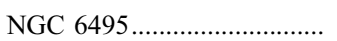 & $1998 \mathrm{bp}$ & 20 & $20^{\prime \prime}$ & 34 \\
\hline 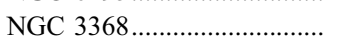 & 1998bu & 45 & $2^{\prime}$ & 57 \\
\hline NGC $0252 \ldots \ldots$ & 1998de & 90 & $30^{\prime \prime}$ & 40 \\
\hline 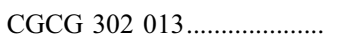 & 1998di & -30 & $0^{\prime \prime}$ & 17 \\
\hline UGC $00139 \ldots \ldots \ldots \ldots \ldots$ & $1998 \mathrm{dk}$ & 80 & $15^{\prime \prime}$ & 51 \\
\hline Anon & $1998 \mathrm{dm}$ & 20 & $15^{\prime \prime}$ & 68 \\
\hline Anon & $1998 d x$ & -30 & $10^{\prime \prime}$ & 29 \\
\hline 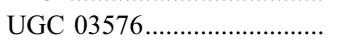 & $1998 \mathrm{ec}$ & 130 & $30^{\prime \prime}$ & 38 \\
\hline UGC 00646 & $1998 \mathrm{ef}$ & 100 & $15^{\prime \prime}$ & 19 \\
\hline 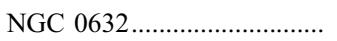 & 1998es & -20 & $\ldots$ & 31 \\
\hline 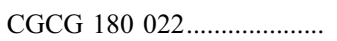 & 1999X & -20 & $15^{\prime \prime}$ & 17 \\
\hline 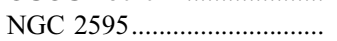 & 1999aa & -20 & $1^{\prime}$ & 57 \\
\hline 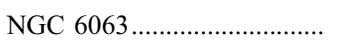 & $1999 \mathrm{ac}$ & -30 & $30^{\prime \prime}$ & 34 \\
\hline NGC $2841 \ldots \ldots$. & 1999by & 65 & $2^{\prime}$ & 51 \\
\hline NGC $6038 \ldots$. & $1999 \mathrm{cc}$ & 90 & $30^{\prime \prime}$ & 50 \\
\hline NGC $6411 \ldots \ldots \ldots \ldots$ & $1999 d a$ & 70 & $20^{\prime \prime}$ & 31 \\
\hline NGC $6951 \ldots \ldots \ldots$ & $2000 \mathrm{e}$ & 0 & $\ldots$ & 32 \\
\hline
\end{tabular}


TABLE 2

EMission-Line EQuivalent Widths (Å)

\begin{tabular}{|c|c|c|c|c|c|c|c|c|c|c|c|}
\hline Galaxy & $\mathrm{SN}$ & $\begin{array}{l}{[\mathrm{O} \text { II }]} \\
\lambda 3727\end{array}$ & $\begin{array}{c}\mathrm{H} \beta \\
\lambda 4861\end{array}$ & $\begin{array}{l}{\left[\begin{array}{ll}\mathrm{O} & \mathrm{III}\end{array}\right]} \\
\lambda 4959\end{array}$ & $\begin{array}{l}{[\mathrm{O} \mathrm{III}]} \\
\lambda 5007\end{array}$ & $\begin{array}{c}{[\mathrm{O} \text { I }]} \\
\lambda 6300\end{array}$ & $\begin{array}{l}{[\mathrm{N} \text { II] }} \\
\lambda 6548\end{array}$ & $\begin{array}{c}\mathrm{H} \alpha \\
\lambda 6562\end{array}$ & $\begin{array}{l}{[\mathrm{N} \text { II] }} \\
\lambda 6584\end{array}$ & $\begin{array}{l}{[\mathrm{S} \text { II }]} \\
\lambda 6717\end{array}$ & $\begin{array}{l}{[\mathrm{S} \text { II] }} \\
\lambda 6731\end{array}$ \\
\hline 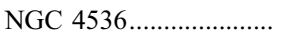 & 1981B & 10.95 & 2.77 & 0.65 & 2.29 & 1.03 & 2.91 & 21.36 & 9.18 & 5.29 & 3.93 \\
\hline 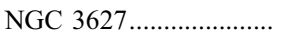 & 1989B & 1.27 & 1.74 & 0.10 & 0.40 & 0.34 & 1.73 & 13.30 & 6.27 & 2.46 & 2.06 \\
\hline 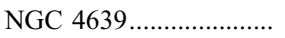 & $1990 \mathrm{~N}$ & 20.76 & 1.39 & 0.29 & 1.65 & 0.88 & 1.14 & 9.34 & 5.23 & 3.00 & 1.76 \\
\hline CGCG $111016 \ldots \ldots \ldots \ldots$ & 19900 & 4.84 & 0.00 & 0.00 & 0.13 & 0.49 & 0.41 & 3.85 & 3.56 & 0.87 & 1.45 \\
\hline NGC $4527 \ldots$ & $1991 \mathrm{~T}$ & 3.20 & 0.60 & 0.18 & 1.78 & 0.00 & 0.85 & 9.29 & 4.70 & 1.62 & 1.66 \\
\hline 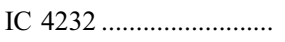 & $1991 U$ & 10.84 & 2.83 & 0.77 & 0.66 & 0.55 & 4.27 & 15.92 & 7.65 & 2.38 & 7.41 \\
\hline 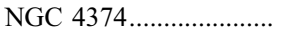 & 1991bg & $\ldots$ & $\ldots$ & $\ldots$ & $\ldots$ & $\ldots$ & 0.00 & 0.04 & 0.44 & $\ldots$ & $\ldots$ \\
\hline 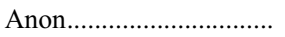 & 1992J & $\ldots$ & $\ldots$ & $\ldots$ & $\ldots$ & $\ldots$ & 0.00 & 0.25 & 0.39 & $\ldots$ & $\ldots$ \\
\hline 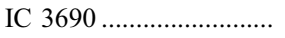 & $1992 \mathrm{P}$ & 13.08 & 0.42 & 0.36 & 1.13 & 1.06 & 1.17 & 7.69 & 5.20 & 2.45 & 2.37 \\
\hline 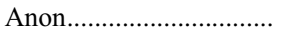 & 1992ag & 42.14 & 9.17 & 3.87 & 13.52 & 0.99 & 7.30 & 39.01 & 12.95 & 12.98 & 6.03 \\
\hline 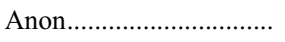 & $1992 b p$ & 8.73 & 0.00 & 0.00 & 3.04 & 0.00 & 1.89 & 7.49 & 5.64 & 5.18 & 6.12 \\
\hline CGCG $307023 \ldots \ldots \ldots \ldots . .$. & $1993 \mathrm{ac}$ & $\ldots$ & $\ldots$ & $\ldots$ & $\ldots$ & $\ldots$ & $\ldots$ & $\ldots$ & $\ldots$ & $\ldots$ & $\ldots$ \\
\hline 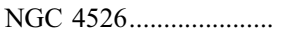 & 1994D & $\ldots$ & $\ldots$ & $\ldots$ & $\ldots$ & $\ldots$ & 0.05 & 0.00 & 0.16 & $\ldots$ & $\ldots$ \\
\hline 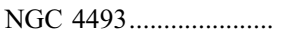 & 1994M & $\ldots$ & $\ldots$ & $\ldots$ & $\ldots$ & $\ldots$ & 0.18 & 0.12 & 0.19 & $\ldots$ & $\ldots$ \\
\hline CGCG $224104 \ldots \ldots \ldots \ldots . . .$. & 1994Q & 25.97 & 5.60 & 0.37 & 2.15 & 0.82 & 2.00 & 24.80 & 6.61 & 6.10 & 3.85 \\
\hline 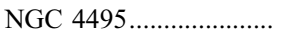 & $1994 \mathrm{~S}$ & 20.37 & 4.37 & 1.22 & 3.93 & 0.73 & 2.09 & 22.33 & 10.01 & 4.42 & 5.88 \\
\hline 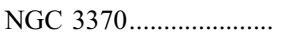 & 1994ae & 16.04 & 3.59 & 0.66 & 0.72 & 0.00 & 2.20 & 19.04 & 7.28 & 4.66 & 3.06 \\
\hline NGC $2962 \ldots \ldots \ldots \ldots \ldots \ldots . . . . . . . .$. & 1995D & $\ldots$ & $\ldots$ & $\ldots$ & $\ldots$ & $\ldots$ & 0.13 & 0.08 & 0.40 & $\ldots$ & $\ldots$ \\
\hline 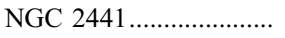 & $1995 \mathrm{E}$ & 12.06 & 2.55 & 0.40 & 1.42 & 0.13 & 1.58 & 11.82 & 5.99 & 3.51 & 2.62 \\
\hline 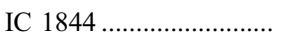 & $1995 \mathrm{ak}$ & 17.56 & 3.98 & 0.43 & 1.81 & 0.60 & 2.39 & 24.07 & 9.18 & 6.57 & 4.42 \\
\hline 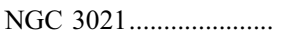 & 1995al & 13.29 & 4.35 & 0.49 & 1.49 & 0.76 & 2.87 & 23.51 & 9.69 & 4.01 & 3.51 \\
\hline UGC $03151 \ldots \ldots \ldots \ldots \ldots \ldots$ & $1995 b d$ & $\ldots$ & $\ldots$ & $\ldots$ & $\ldots$ & $\ldots$ & 0.78 & 4.36 & 4.78 & $\ldots$ & $\ldots$ \\
\hline 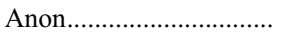 & 1996C & 17.18 & 2.37 & 0.00 & 1.46 & 0.80 & 4.38 & 14.27 & 7.75 & 5.54 & 3.57 \\
\hline 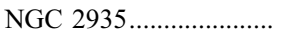 & $1996 Z$ & $\ldots$ & $\ldots$ & $\ldots$ & $\ldots$ & $\ldots$ & 0.98 & 4.01 & 3.42 & $\ldots$ & $\ldots$ \\
\hline Anon................................. & $1996 \mathrm{ab}$ & $\ldots$ & $\ldots$ & $\ldots$ & $\cdots$ & $\ldots$ & 0.61 & 3.71 & 2.87 & $\ldots$ & $\ldots$ \\
\hline 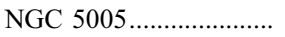 & 1996ai & $\ldots$ & $\ldots$ & $\ldots$ & $\ldots$ & $\ldots$ & 0.89 & 3.66 & 3.57 & $\ldots$ & $\ldots$ \\
\hline Anon............................... & 1996bl & 3.66 & 0.48 & 0.30 & 0.85 & 0.84 & 2.25 & 9.56 & 5.53 & 3.24 & 2.27 \\
\hline NGC $0673 \ldots \ldots \ldots \ldots \ldots \ldots . . . . . . . . .$. & 1996bo & 20.42 & 6.34 & 1.98 & 3.52 & 0.59 & 3.40 & 29.89 & 11.20 & 6.30 & 5.31 \\
\hline 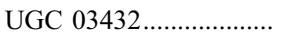 & $1996 \mathrm{bv}$ & 16.45 & 2.40 & 0.83 & 4.47 & 0.83 & 1.39 & 13.58 & 3.37 & 4.88 & 4.30 \\
\hline 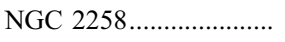 & $1997 \mathrm{E}$ & $\ldots$ & $\ldots$ & $\ldots$ & $\ldots$ & $\ldots$ & $\ldots$ & $\ldots$ & $\ldots$ & $\ldots$ & $\ldots$ \\
\hline 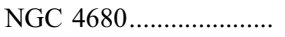 & 1997bp & 7.13 & 3.81 & 0.10 & 1.48 & 0.21 & 2.52 & 21.55 & 9.43 & 4.37 & 3.32 \\
\hline 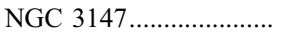 & $1997 \mathrm{bq}$ & $\ldots$ & $\ldots$ & $\ldots$ & $\ldots$ & $\ldots$ & 0.83 & 7.15 & 4.08 & $\ldots$ & $\ldots$ \\
\hline Anon.................................. & $1997 \mathrm{br}$ & 41.89 & 5.65 & 2.27 & 13.02 & 1.70 & 3.55 & 30.09 & 9.74 & 4.11 & 3.89 \\
\hline NGC $5490 \ldots \ldots \ldots \ldots \ldots \ldots . . . . . . . .$. & $1997 \mathrm{cn}$ & $\ldots$ & $\ldots$ & $\ldots$ & $\ldots$ & $\ldots$ & 0.03 & 0.03 & 0.03 & $\ldots$ & $\ldots$ \\
\hline 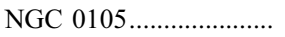 & $1997 \mathrm{cw}$ & 17.21 & 3.79 & 2.11 & 5.67 & 0.75 & 3.74 & 23.67 & 9.71 & 5.15 & 4.08 \\
\hline UGC $03845 \ldots \ldots \ldots \ldots \ldots \ldots$ & 1997do & 31.51 & 4.81 & 1.03 & 5.44 & 0.53 & 1.36 & 22.70 & 6.04 & 6.00 & 4.71 \\
\hline NGC $5440 \ldots \ldots \ldots \ldots \ldots \ldots . . . . . . . .$. & 1998D & $\ldots$ & $\ldots$ & $\ldots$ & $\ldots$ & $\ldots$ & 0.32 & 0.11 & 1.93 & $\ldots$ & $\ldots$ \\
\hline 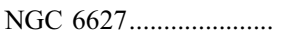 & $1998 \mathrm{~V}$ & 7.25 & 2.58 & 0.20 & 3.86 & 0.42 & 2.73 & 14.03 & 9.03 & 2.85 & 2.20 \\
\hline 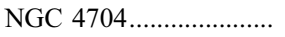 & $1998 \mathrm{ab}$ & 11.00 & 2.93 & 0.23 & 0.70 & 0.27 & 2.67 & 10.29 & 7.54 & 4.98 & 1.38 \\
\hline 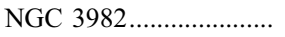 & $1998 \mathrm{aq}$ & 17.40 & 5.47 & 0.91 & 3.32 & 0.65 & 3.30 & 27.44 & 11.15 & 5.81 & 3.98 \\
\hline 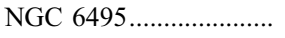 & 1998bp & $\ldots$ & $\ldots$ & $\ldots$ & $\ldots$ & $\ldots$ & 0.09 & 0.02 & 0.15 & $\ldots$ & $\ldots$ \\
\hline 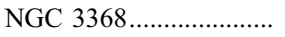 & $1998 b u$ & $\ldots$ & $\ldots$ & $\ldots$ & $\ldots$ & $\ldots$ & 0.64 & 1.04 & 1.92 & $\ldots$ & $\ldots$ \\
\hline 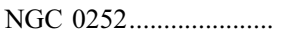 & 1998de & $\cdots$ & $\ldots$ & $\ldots$ & $\cdots$ & $\ldots$ & 0.28 & 0.45 & 1.79 & $\ldots$ & $\ldots$ \\
\hline CGCG $302013 \ldots \ldots \ldots \ldots . . .$. & 1998di & $\ldots$ & $\ldots$ & $\ldots$ & $\ldots$ & $\ldots$ & 0.31 & 0.31 & 0.31 & $\ldots$ & $\ldots$ \\
\hline UGC $00139 \ldots \ldots \ldots \ldots \ldots \ldots$ & $1998 \mathrm{dk}$ & 13.07 & 4.59 & 0.62 & 2.48 & 0.67 & 1.86 & 21.06 & 7.18 & 5.45 & 3.91 \\
\hline UGCA $017 \ldots \ldots \ldots \ldots \ldots \ldots$ & $1998 \mathrm{dm}$ & 30.67 & 5.43 & 2.42 & 9.19 & 0.83 & 1.38 & 26.79 & 5.32 & 6.92 & 4.60 \\
\hline 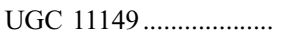 & $1998 \mathrm{dx}$ & $\ldots$ & $\ldots$ & $\ldots$ & $\ldots$ & $\ldots$ & 0.14 & 0.14 & 0.14 & $\ldots$ & $\ldots$ \\
\hline UGC $03576 \ldots \ldots \ldots \ldots \ldots . . . . . .$. & $1998 \mathrm{ec}$ & 10.80 & 0.00 & 0.08 & 0.14 & 0.50 & 1.00 & 4.41 & 3.66 & 1.64 & 4.20 \\
\hline UGC $00646 \ldots \ldots \ldots \ldots \ldots \ldots$ & $1998 \mathrm{ef}$ & $\ldots$ & $\ldots$ & $\ldots$ & $\ldots$ & $\ldots$ & 0.08 & 0.00 & 0.87 & $\ldots$ & $\ldots$ \\
\hline NGC $0632 \ldots \ldots \ldots \ldots \ldots \ldots . . . . . . . .$. & $1998 \mathrm{es}$ & 21.55 & 6.82 & 0.74 & 3.03 & 1.60 & 3.89 & 31.99 & 13.11 & 6.87 & 5.38 \\
\hline CGCG $180022 \ldots \ldots \ldots \ldots . .$. & 1999X & $\ldots$ & $\ldots$ & $\ldots$ & $\ldots$ & $\ldots$ & 0.25 & 0.60 & 0.70 & $\ldots$ & $\ldots$ \\
\hline 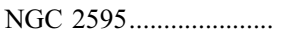 & 1999aа & 15.35 & 0.54 & 0.26 & 0.42 & 0.00 & 1.55 & 11.88 & 6.66 & 3.17 & 2.91 \\
\hline 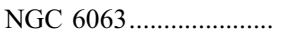 & $1999 \mathrm{ac}$ & 9.16 & 2.56 & 0.00 & 0.78 & 0.46 & 1.27 & 11.24 & 5.22 & 2.71 & 4.22 \\
\hline 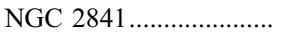 & 1999by & $\ldots$ & $\ldots$ & $\ldots$ & $\ldots$ & $\ldots$ & 0.11 & 0.74 & 1.65 & $\ldots$ & $\ldots$ \\
\hline 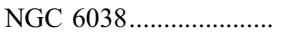 & $1999 \mathrm{cc}$ & $\ldots$ & $\ldots$ & $\ldots$ & $\ldots$ & $\ldots$ & 1.53 & 9.50 & 4.54 & $\ldots$ & $\ldots$ \\
\hline 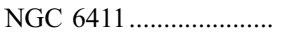 & $1999 \mathrm{da}$ & $\ldots$ & $\ldots$ & $\ldots$ & $\ldots$ & $\ldots$ & 0.02 & 0.00 & 0.06 & $\ldots$ & $\ldots$ \\
\hline 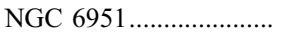 & $2000 \mathrm{E}$ & $\ldots$ & $\ldots$ & $\ldots$ & $\ldots$ & $\ldots$ & 1.26 & 3.68 & 2.84 & $\ldots$ & $\ldots$ \\
\hline
\end{tabular}


TABLE 3

Emission-Line FluXes $\left(10^{14} \mathrm{ergs}^{-2} \mathrm{~s}^{-1}\right)$

\begin{tabular}{|c|c|c|c|c|c|c|c|c|c|c|c|}
\hline Galaxy & $\mathrm{SN}$ & $\begin{array}{l}{[\mathrm{O} \text { II] }} \\
\lambda 3727\end{array}$ & $\begin{array}{c}\mathrm{H} \beta \\
\lambda 4861\end{array}$ & $\begin{array}{l}{[\mathrm{O} \text { III] }} \\
\lambda 4959\end{array}$ & $\begin{array}{l}{[\mathrm{O} \text { III] }} \\
\lambda 5007\end{array}$ & $\begin{array}{c}{[\mathrm{O} \mathrm{I}]} \\
\lambda 6300\end{array}$ & $\begin{array}{l}{[\mathrm{N} \text { II] }} \\
\lambda 6548\end{array}$ & $\begin{array}{c}\mathrm{H} \alpha \\
\lambda 6562\end{array}$ & $\begin{array}{l}{[\mathrm{N} \text { II }]} \\
\lambda 6584\end{array}$ & $\begin{array}{c}{[\mathrm{S} \text { II }]} \\
\lambda 6717\end{array}$ & $\begin{array}{l}{[\mathrm{S} \text { II] }} \\
\lambda 6731\end{array}$ \\
\hline 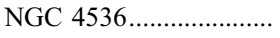 & 1981B & 3.11 & 0.76 & 0.20 & 0.68 & 0.30 & 0.86 & 6.32 & 2.72 & 1.58 & 1.18 \\
\hline NGC $3627 \ldots \ldots \ldots \ldots \ldots \ldots$ & 1989B & 1.22 & 2.14 & 0.14 & 0.52 & 0.38 & 1.97 & 15.10 & 7.14 & 2.81 & 2.35 \\
\hline 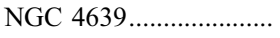 & $1990 \mathrm{~N}$ & 4.94 & 0.60 & 0.13 & 0.71 & 0.34 & 0.45 & 3.68 & 2.05 & 1.19 & 0.70 \\
\hline CGCG $111016 \ldots \ldots \ldots \ldots . . . .$. & 19900 & 0.37 & 0.00 & 0.00 & 0.02 & 0.06 & 0.06 & 0.52 & 0.47 & 0.11 & 0.18 \\
\hline 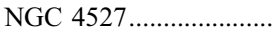 & $1991 \mathrm{~T}$ & 0.84 & 0.38 & 0.09 & 0.59 & 0.00 & 0.35 & 3.76 & 1.90 & 0.64 & 0.65 \\
\hline 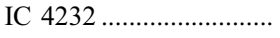 & $1991 \mathrm{U}$ & 0.10 & 0.60 & 0.13 & 0.13 & 0.08 & 0.72 & 2.68 & 1.29 & 0.40 & 1.24 \\
\hline 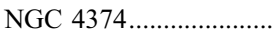 & $1991 \mathrm{bg}$ & $\ldots$ & $\ldots$ & $\ldots$ & $\ldots$ & $\ldots$ & $\ldots$ & 0.03 & 0.32 & $\ldots$ & $\ldots$ \\
\hline 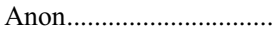 & $1992 J$ & $\ldots$ & $\ldots$ & $\ldots$ & $\ldots$ & $\ldots$ & $\ldots$ & 0.04 & 0.07 & $\ldots$ & $\ldots$ \\
\hline IC $3690 \ldots \ldots . .$. & $1992 \mathrm{P}$ & 0.59 & 0.04 & 0.04 & 0.12 & 0.10 & 0.12 & 0.79 & 0.53 & 0.24 & 0.24 \\
\hline 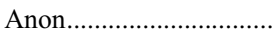 & 1992ag & 2.98 & 1.14 & 0.54 & 1.71 & 0.11 & 0.87 & 4.70 & 1.60 & 1.64 & 0.74 \\
\hline 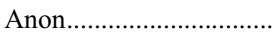 & $1992 b p$ & 0.18 & 0.00 & 0.00 & 0.09 & 0.00 & 0.05 & 0.20 & 0.15 & 0.13 & 0.15 \\
\hline CGCG $307023 \ldots \ldots \ldots \ldots . .$. & $1993 \mathrm{ac}$ & $\ldots$ & $\ldots$ & $\ldots$ & $\ldots$ & $\ldots$ & $\ldots$ & $\ldots$ & $\ldots$ & $\ldots$ & $\ldots$ \\
\hline NGC $4526 \ldots \ldots \ldots \ldots \ldots \ldots \ldots$ & 1994D & $\ldots$ & $\ldots$ & $\ldots$ & $\ldots$ & $\cdots$ & 0.06 & $\ldots$ & 0.20 & $\ldots$ & $\ldots$ \\
\hline 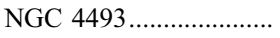 & 1994M & $\ldots$ & $\ldots$ & $\ldots$ & $\ldots$ & $\ldots$ & 0.03 & 0.16 & 0.03 & $\ldots$ & $\ldots$ \\
\hline CGCG 224 104 ............. & 1994Q & 0.84 & 0.27 & 0.02 & 0.12 & 0.04 & 0.09 & 1.09 & 0.29 & 0.27 & 0.17 \\
\hline 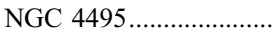 & $1994 \mathrm{~S}$ & 2.90 & 0.97 & 0.31 & 0.92 & 0.18 & 0.49 & 5.25 & 2.35 & 1.03 & 1.37 \\
\hline 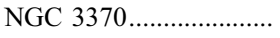 & $1994 \mathrm{ae}$ & 2.22 & 0.65 & 0.21 & 0.14 & 0.00 & 0.16 & 3.19 & 1.21 & 0.75 & 0.49 \\
\hline NGC $2962 \ldots \ldots . .$. & 1995D & $\ldots$ & $\ldots$ & $\ldots$ & $\ldots$ & $\ldots$ & 0.04 & 0.02 & 0.13 & $\ldots$ & $\ldots$ \\
\hline NGC $2441 \ldots \ldots \ldots . .$. & $1995 \mathrm{E}$ & 1.47 & 0.44 & 0.08 & 0.26 & 0.02 & 0.25 & 1.88 & 0.95 & 0.54 & 0.40 \\
\hline 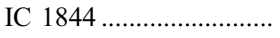 & 1995ak & 1.91 & 0.64 & 0.08 & 0.31 & 0.09 & 0.37 & 3.69 & 1.41 & 0.98 & 0.66 \\
\hline 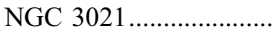 & 1995al & 3.79 & 1.93 & 0.24 & 0.69 & 0.31 & 1.19 & 9.76 & 4.02 & 1.64 & 1.44 \\
\hline UGC $03151 \ldots \ldots \ldots \ldots \ldots \ldots \ldots$ & 1995bd & $\ldots$ & $\ldots$ & $\ldots$ & $\cdots$ & $\ldots$ & 0.47 & 2.59 & 2.80 & $\ldots$ & $\ldots$ \\
\hline 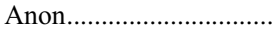 & $1996 \mathrm{C}$ & 0.57 & 0.13 & 0.00 & 0.08 & 0.04 & 0.22 & 0.70 & 0.37 & 0.27 & 0.17 \\
\hline 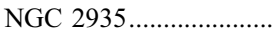 & $1996 Z$ & $\ldots$ & $\ldots$ & $\ldots$ & $\ldots$ & $\ldots$ & 0.29 & 1.17 & 0.98 & $\ldots$ & $\ldots$ \\
\hline 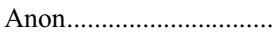 & $1996 \mathrm{ab}$ & $\ldots$ & $\ldots$ & $\ldots$ & $\ldots$ & $\ldots$ & 0.04 & 0.22 & 0.16 & $\ldots$ & $\ldots$ \\
\hline NGC $5005 \ldots \ldots \ldots \ldots \ldots \ldots . . . . . . .$. & 1996ai & $\ldots$ & $\ldots$ & $\ldots$ & $\ldots$ & $\ldots$ & 0.89 & 3.67 & 3.58 & 1.71 & 0.90 \\
\hline 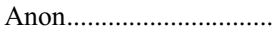 & 1996bl & 0.16 & 0.03 & 0.02 & 0.06 & 0.05 & 0.14 & 0.58 & 0.34 & 0.18 & 0.13 \\
\hline 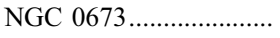 & 1996bo & 3.93 & 1.46 & 0.51 & 0.89 & 0.12 & 0.70 & 6.13 & 2.29 & 1.26 & 1.06 \\
\hline UGC $03432 \ldots \ldots \ldots \ldots \ldots \ldots$ & 1996bv & 2.32 & 0.40 & 0.16 & 0.75 & 0.12 & 0.21 & 2.11 & 0.53 & 0.73 & 0.65 \\
\hline 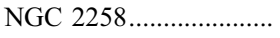 & $1997 \mathrm{E}$ & $\ldots$ & $\ldots$ & $\ldots$ & $\ldots$ & $\ldots$ & $\ldots$ & $\ldots$ & $\ldots$ & $\ldots$ & $\ldots$ \\
\hline 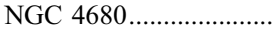 & 1997bp & 1.92 & 1.15 & 0.03 & 0.47 & 0.06 & 0.75 & 6.42 & 2.81 & 1.30 & 0.99 \\
\hline 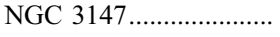 & $1997 \mathrm{bq}$ & $\ldots$ & $\ldots$ & $\ldots$ & $\ldots$ & $\ldots$ & 0.43 & 3.66 & 2.07 & $\ldots$ & $\ldots$ \\
\hline 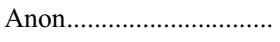 & $1997 \mathrm{br}$ & 3.13 & 0.89 & 0.48 & 2.24 & 0.24 & 0.41 & 3.51 & 1.15 & 0.62 & 0.60 \\
\hline 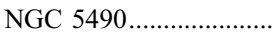 & $1997 \mathrm{cn}$ & $\ldots$ & $\ldots$ & $\ldots$ & $\ldots$ & $\ldots$ & 0.01 & 0.01 & 0.01 & $\ldots$ & $\ldots$ \\
\hline 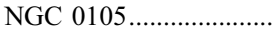 & $1997 \mathrm{cw}$ & 2.52 & 0.72 & 0.43 & 1.09 & 0.12 & 0.60 & 3.82 & 1.56 & 0.81 & 0.63 \\
\hline UGC $03845 \ldots \ldots \ldots \ldots \ldots \ldots$ & 1997do & 4.79 & 0.93 & 0.23 & 1.11 & 0.09 & 0.22 & 3.68 & 0.98 & 0.95 & 0.74 \\
\hline NGC $5440 \ldots \ldots \ldots \ldots \ldots \ldots . . . . . . . .$. & 1998D & $\ldots$ & $\ldots$ & $\ldots$ & $\ldots$ & $\ldots$ & 0.11 & 0.04 & 0.64 & $\ldots$ & $\ldots$ \\
\hline 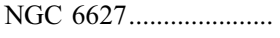 & 1998V & 1.65 & 0.92 & 0.08 & 1.46 & 0.16 & 1.07 & 5.51 & 3.55 & 1.12 & 0.87 \\
\hline 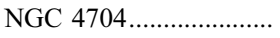 & $1998 \mathrm{ab}$ & 0.62 & 0.34 & 0.03 & 0.09 & 0.03 & 0.32 & 1.23 & 0.88 & 0.62 & 0.17 \\
\hline 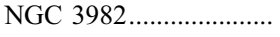 & $1998 \mathrm{aq}$ & 5.93 & 2.53 & 0.46 & 1.64 & 0.26 & 1.33 & 11.10 & 4.46 & 2.29 & 1.57 \\
\hline 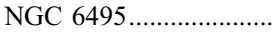 & 1998bp & $\ldots$ & $\ldots$ & $\ldots$ & $\ldots$ & $\ldots$ & 0.04 & 0.01 & 0.06 & $\ldots$ & $\ldots$ \\
\hline NGC $3368 \ldots \ldots \ldots \ldots \ldots \ldots . . . . . . . .$. & 1998bu & $\ldots$ & $\ldots$ & $\ldots$ & $\ldots$ & $\ldots$ & 0.84 & 1.37 & 2.52 & $\ldots$ & $\cdots$ \\
\hline NGC $0252 \ldots \ldots \ldots \ldots \ldots \ldots . . . . . . . .$. & $1998 \mathrm{de}$ & $\ldots$ & $\ldots$ & $\ldots$ & $\ldots$ & $\ldots$ & 0.13 & 0.20 & 0.80 & $\ldots$ & $\ldots$ \\
\hline CGCG $302013 \ldots \ldots \ldots \ldots . .$. & 1998di & $\ldots$ & $\ldots$ & $\ldots$ & $\ldots$ & $\ldots$ & 0.01 & 0.01 & 0.01 & $\ldots$ & $\ldots$ \\
\hline UGC $00139 \ldots \ldots \ldots \ldots \ldots \ldots$ & $1998 \mathrm{dk}$ & 2.34 & 0.85 & 0.21 & 0.50 & 0.17 & 0.32 & 3.59 & 1.22 & 0.92 & 0.66 \\
\hline UGCA $017 \ldots \ldots \ldots \ldots \ldots \ldots . . . . . . . .$. & $1998 \mathrm{dm}$ & 9.28 & 1.89 & 0.97 & 3.46 & 0.25 & 0.41 & 7.84 & 1.55 & 1.98 & 1.31 \\
\hline 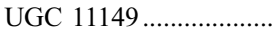 & $1998 \mathrm{dx}$ & $\ldots$ & $\ldots$ & $\ldots$ & $\ldots$ & $\ldots$ & 0.01 & 0.01 & 0.01 & $\ldots$ & $\ldots$ \\
\hline UGC $03576 \ldots \ldots \ldots \ldots \ldots . . . . . . .$. & $1998 \mathrm{ec}$ & 0.89 & 0.00 & 0.01 & 0.02 & 0.08 & 0.16 & 0.71 & 0.59 & 0.26 & 0.64 \\
\hline UGC $00646 \ldots \ldots \ldots \ldots \ldots \ldots$ & 1998ef & $\ldots$ & $\ldots$ & $\ldots$ & $\ldots$ & $\cdots$ & 0.02 & 0.00 & 0.22 & $\ldots$ & $\ldots$ \\
\hline NGC $0632 \ldots \ldots \ldots \ldots \ldots \ldots \ldots$ & 1998es & 3.14 & 1.53 & 0.19 & 0.71 & 0.30 & 0.76 & 6.20 & 2.53 & 1.29 & 1.01 \\
\hline CGCG $180022 \ldots \ldots \ldots \ldots . .$. & 1999X & $\ldots$ & $\ldots$ & $\ldots$ & $\ldots$ & $\ldots$ & 0.01 & 0.03 & 0.03 & $\ldots$ & $\ldots$ \\
\hline 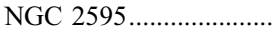 & 1999aа & 2.05 & 0.14 & 0.07 & 0.10 & 0.00 & 0.37 & 2.73 & 1.48 & 0.73 & 0.67 \\
\hline NGC $6063 \ldots \ldots \ldots \ldots \ldots . . . . . . . . .$. & $1999 \mathrm{ac}$ & 1.30 & 0.42 & 0.00 & 0.14 & 0.07 & 0.20 & 1.73 & 0.80 & 0.41 & 0.64 \\
\hline NGC $2841 \ldots \ldots \ldots \ldots \ldots \ldots \ldots$ & 1999by & $\ldots$ & $\ldots$ & $\ldots$ & $\ldots$ & $\cdots$ & 0.09 & 0.56 & 1.25 & $\ldots$ & $\cdots$ \\
\hline 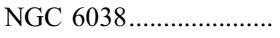 & $1999 \mathrm{cc}$ & $\ldots$ & $\ldots$ & $\ldots$ & $\ldots$ & $\ldots$ & 0.23 & 1.44 & 0.70 & $\ldots$ & $\ldots$ \\
\hline 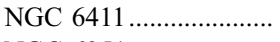 & 1999 da & $\cdots$ & $\cdots$ & $\ldots$ & $\cdots$ & $\cdots$ & 0.01 & 0.00 & 0.03 & $\ldots$ & $\ldots$ \\
\hline 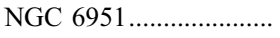 & $2000 \mathrm{E}$ & $\ldots$ & $\ldots$ & $\ldots$ & $\ldots$ & $\ldots$ & 0.91 & 2.63 & 2.00 & $\ldots$ & $\ldots$ \\
\hline
\end{tabular}


Following the flux calibration, a telluric absorption correction was performed on those galaxy spectra containing relevant emission lines (i.e., $\mathrm{H} \alpha$ and $\mathrm{S}$ II) that have been redshifted into the $B$ band (6860-6890 $\AA$ ) and beyond. Next, the spectra were dereddened to account for local reddening due to Galactic extinction. This was done using the routine deredden in IRAF. In each case a value of 3.0 was taken for the total to selective visual absorption ratio, $R$. Furthermore, the value of the color excess $E(B-V)$ was chosen for each galaxy direction to correspond to the one stated by the NASA/IPAC Extragalactic Database (NED). These color excess values were calculated from $C O B E$, the IRAS maps, and the Leiden-Dwingeloo maps of $\mathrm{H}_{\mathrm{I}}$ emission. Finally, the galaxy spectra were Doppler-corrected using the routine dopcor, with redshifts obtained from NED.

\subsection{Line Strengths}

Following reduction, the spectral properties were analyzed through the identification and subsequent line profiling of various relevant spectral lines. In each case the line strengths were recorded using the splot routine in IRAF. Gaussian line profiles were fit for each emission line individually, with the primary source of error originating in the continuum definition. If appropriate, a boxcar-smoothing algorithm was applied interactively, allowing for more accurate continuum definition. We obtained both equivalent width (EW) and line fluxes for [O II] $\lambda 3727$ (our resolution was insufficient to resolve the [O $\mathrm{II}]$ doublet), $\mathrm{H} \beta \lambda 4861$, [O III] $\lambda 4959$, [O III] $\lambda 5007$, [O I] $\lambda 6300$, [N II] $\lambda 6548$, $\mathrm{H} \alpha \lambda 6562,[\mathrm{~N}$ II] $\lambda 6584$, [S II] $\lambda 6717$, and [S II] $\lambda 6731$. The EWs measured in angstroms are shown in Table 2, while emissionline fluxes in units of $10^{-14} \mathrm{ergs} \mathrm{cm}^{-2} \mathrm{~s}^{-1}$ are given in Table 3, respectively.

\section{RESULTS}

\subsection{Host Galaxy and Supernova Parameterization}

Here we describe the parameters that characterize the galaxies in the SN Ia host sample. The galactic parameters are given in Table 4. Columns (3)-(6) are observed, while columns (7) and (8) are derived parameters. Column (1) lists the galaxies in our sample, while column (2) gives each galaxy's hosted supernova. The absolute $B$ magnitudes of each host along with their corresponding errors are recorded in columns (3) and (4), respectively. The vast majority of magnitudes were calculated from distances derived from their respective redshifts. In a few cases, the potential for uncertainty was heightened due to the low recessional velocity of the host galaxy. Therefore, other distance measurements from the literature were employed for these cases when possible. Cepheid-based distance moduli were found for NGC 3368, NGC 3627, NGC 4639, and NGC 4536 from the HST Key Project published in Gibson et al. (2000). The distance to NGC 4526 was determined by Hamuy et al. (1996a) using the surface brightness fluctuations-planetary nebula luminosity function published in Hamuy et al. (2000, hereafter H00). All magnitudes were corrected to correspond to a Hubble constant of $72 \mathrm{~km} \mathrm{~s}^{-1} \mathrm{Mpc}^{-1}$. Column (5) lists the morphological types according to NED, while column (6) shows the $\mathrm{H} \alpha$ luminosity for each host galaxy.

The derived galactic parameters were metallicity and birthrate parameter $b$ (Scalo 1986). These are shown in columns (7) and (8), respectively. For those host galaxies with distinguishable emission lines, we determined the metallicities from our emissionline flux measurements using the models detailed in Kewley \& Dopita (2002). The paper provides a series of line strength diagnostic diagrams with various dependences on both metallicity and the local ionization parameter, $q$. One first estimates an initial metallicity through a diagnostic that varies little with $q$. The initial value is then used to pin down the value of the ionization parameter through a diagnostic with strong dependences on both metallicity and the ionization parameter. Successive iterations ultimately provide the best estimate of the galaxy's metallicity. For full details see Kewley \& Dopita (2002). Extinction correction was applied for those galaxies with measurable Balmer emission using the Whitford reddening curve as parameterized by Miller \& Mathews (1972). We were unsuccessful in obtaining metallicity estimates for galaxies with weak emission. Furthermore, our signalto-noise ratio was insufficient to provide accurate absorption-line strengths needed for an absorption-line metallicity estimate. However, three galaxies from our sample had metallicities measured in H00, which we used in our analysis.

The final host galaxy parameter was the Scalo $b$ parameter (Scalo 1986). The Scalo $b$ parameter is the ratio of the current SFR to the average SFR of the past. The parameter was determined by interpolation of the plot given in Figure 3 from Kennicutt et al. (1994, hereafter KTC94). The plot shows the dependence of $b$ on $\mathrm{EW}(\mathrm{H} \alpha+[\mathrm{N}$ II $])$ as dictated by the exponential-plus-burst model detailed in KTC94.

Our SNe were characterized according to the parameters shown in Table 5. Once again, columns (1) and (2) give the galaxy identification and SN name, respectively. Columns (3) and (4) give the decline rate parameter $\Delta m_{15}(B)$ and its corresponding error, respectively. The parameter $\Delta m_{15}(B)$ is defined as the change in apparent magnitude from maximum to 15 days after maximum light in the supernova rest frame. It acts as a convenient, reddening free, indicator of luminosity. Finally, column (5) shows the Hubble residuals for the SN set. Assuming that Type Ia supernovae are perfect standard candles, the extinction- and light-curve-corrected absolute magnitudes for the SNe should be identical. The Hubble residual for an individual $\mathrm{SN}$ is then defined as the deviation of its light-curve- and color-corrected absolute magnitude from the average light-curve-corrected magnitude in the SN set. Our magnitudes originate from the set of 80 Hubble flow SNe published in Jha (2002).

\subsection{SNe Ia and Host Galaxy Correlations}

It is the goal of this study to investigate the correlations between Type Ia properties and their global host galaxy parameters. Some of these correlations have been explored observationally in the past. Hamuy et al. (1996a) reported that the most luminous SNe Ia tend to be hosted by late-type, spiral galaxies. The same behavior is seen in Figure 1, in which we have replicated the morphological classification versus decline rate plot of Hamuy et al. (1996a) for a large sample of host galaxies. The data were compiled from the SNe described in Phillips et al. (1999), Jha (2002), Riess et al. (1999), and Krisciunas et al. (2004). The vertical lines in Figure 1 represent the average decline rates for the $\mathrm{SNe}$ in late- and earlytype galaxies, respectively. They confirm the results of Hamuy et al., that the slower declining, more luminous supernovae are hosted by late-type galaxies. However, it is important to note when grouping host galaxies by their morphological type that such a grouping does not necessarily imply the members of a common class possess similar physical characteristics, such as metallicity and star formation histories (SFHs). For example, Figure 1 highlights NGC 2841 and NGC 0632, which are categorized as Sa and S0 galaxies, respectively. However, NGC 2841 is a galaxy with none of the emission features typically observed in spiral galaxies. Moreover, NGC 0632, although tentatively labeled an S0 galaxy by NED, shows strong emission indicative of a starburst galaxy. This shows that the gross morphology provides a 
TABLE 4

Galaxy Characterization

\begin{tabular}{|c|c|c|c|c|c|c|c|}
\hline $\begin{array}{c}\text { Galaxy } \\
\text { (1) }\end{array}$ & $\begin{array}{l}\mathrm{SN} \\
(2)\end{array}$ & $\begin{array}{c}M_{B} \\
(\mathrm{mag}) \\
(3)\end{array}$ & $\begin{array}{c}\delta M_{B} \\
(\mathrm{mag}) \\
(4)\end{array}$ & $\begin{array}{l}\text { Morphological } \\
\text { Type } \\
\text { (5) }\end{array}$ & $\begin{array}{c}\text { H } \alpha \text { Luminosity } \\
\left(\text { ergs s }^{-1}\right) \\
(6)\end{array}$ & $\begin{array}{c}\log (\mathrm{O} / \mathrm{H})+12 \\
(7)\end{array}$ & $\begin{array}{c}\text { Scalo } b \\
(8)\end{array}$ \\
\hline 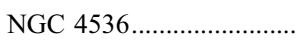 & 1981B & $-19.86^{\mathrm{a}}$ & 0.11 & Sbc & $6.87 \mathrm{E}+39$ & 8.66 & 1.11 \\
\hline 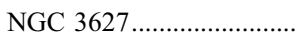 & 1989B & $-20.5^{\mathrm{a}}$ & 0.24 & $\mathrm{Sb}$ & $4.08 \mathrm{E}+39$ & 9.13 & 0.57 \\
\hline 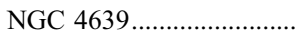 & $1990 \mathrm{~N}$ & $-19.7^{\mathrm{a}}$ & 0.23 & Sbc & $1.49 \mathrm{E}+39$ & 8.76 & 0.37 \\
\hline CGCG $111016 \ldots \ldots \ldots \ldots \ldots$ & 19900 & $\ldots$ & $\ldots$ & Sba & $1.07 \mathrm{E}+40$ & $\ldots$ & 0.15 \\
\hline 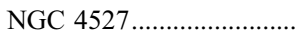 & $1991 \mathrm{~T}$ & -20.5 & 0.27 & Sbc & $3.87 \mathrm{E}+39$ & 8.83 & 0.35 \\
\hline IC $4232 \ldots \ldots \ldots \ldots \ldots \ldots \ldots \ldots$ & $1991 U$ & -21.02 & 0.32 & $\mathrm{Sbc}$ & $6.42 \mathrm{E}+40$ & 9.34 & 0.82 \\
\hline 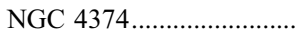 & $1991 b g$ & -21.33 & 0.07 & E1 & $9.42 \mathrm{E}+36$ & $\ldots$ & 0.01 \\
\hline 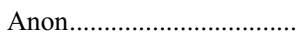 & 1992J & -21.28 & 0.14 & $\mathrm{~S} 0+$ & $1.79 \mathrm{E}+39$ & $\ldots$ & 0.01 \\
\hline IC $3690 \ldots \ldots \ldots \ldots \ldots \ldots \ldots \ldots \ldots$ & $1992 \mathrm{P}$ & -19.91 & 0.27 & $\mathrm{Sb}$ & $1.20 \mathrm{E}+40$ & $\ldots$ & 0.32 \\
\hline 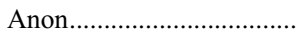 & $1992 \mathrm{ag}$ & $\ldots$ & $\ldots$ & $\mathrm{S}^{\mathrm{b}}$ & $6.97 \mathrm{E}+40$ & 8.87 & 2.05 \\
\hline 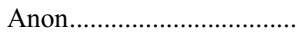 & 1992bp & -20.7 & 0.12 & $\mathrm{E} 2 / \mathrm{S} 0$ & $2.89 \mathrm{E}+40$ & $\ldots$ & 0.35 \\
\hline CGCG $307023 \ldots \ldots \ldots \ldots \ldots$ & $1993 \mathrm{ac}$ & $\ldots$ & $\ldots$ & $\mathrm{E}$ & $\ldots$ & $\ldots$ & 0.01 \\
\hline 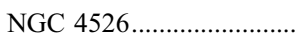 & 1994D & $-20.29^{c}$ & 0.10 & S0 & $\ldots$ & $\ldots$ & 0.01 \\
\hline 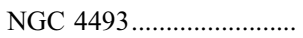 & 1994M & $\ldots$ & $\ldots$ & E+pec & $2.01 \mathrm{E}+39$ & $\ldots$ & 0.01 \\
\hline CGCG 224 104................ & 1994Q & $\ldots$ & $\ldots$ & So & $1.99 \mathrm{E}+40$ & 8.76 & 1.11 \\
\hline 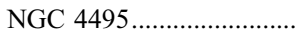 & $1994 \mathrm{~S}$ & -19.94 & 0.30 & $\mathrm{Sab}$ & $2.90 \mathrm{E}+40$ & 8.85 & 1.15 \\
\hline 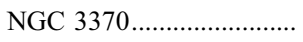 & $1994 \mathrm{ae}$ & -18.96 & 0.28 & $\mathrm{Sc}$ & $1.94 \mathrm{E}+39$ & 8.79 & 0.95 \\
\hline 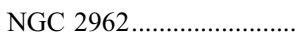 & 1995D & -19.83 & 0.28 & $\mathrm{Sa}$ & $2.94 \mathrm{E}+37$ & $\ldots$ & 0.01 \\
\hline 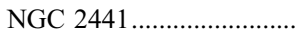 & $1995 \mathrm{E}$ & -20.77 & 0.28 & $\mathrm{Sb}$ & $5.34 \mathrm{E}+39$ & 8.89 & 0.50 \\
\hline 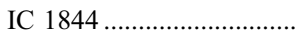 & $1995 \mathrm{ak}$ & $\ldots$ & $\ldots$ & Sbc & $3.79 \mathrm{E}+40$ & 8.79 & 1.19 \\
\hline 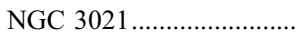 & 1995al & -18.74 & 0.30 & Sbc & $7.36 \mathrm{E}+39$ & 8.94 & 1.20 \\
\hline 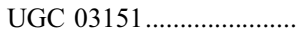 & $1995 b d$ & $\ldots$ & $\ldots$ & So & $1.27 \mathrm{E}+40$ & $\ldots$ & 0.20 \\
\hline 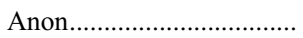 & $1996 \mathrm{C}$ & $\ldots$ & $\ldots$ & $\mathrm{Sa}$ & $1.16 \mathrm{E}+40$ & 8.78 & 0.76 \\
\hline 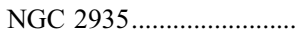 & $1996 Z$ & -20.6 & 0.29 & $\mathrm{Sb}$ & $1.86 \mathrm{E}+39$ & $\ldots$ & 0.17 \\
\hline 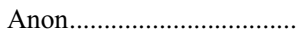 & $1996 \mathrm{ab}$ & $\ldots$ & $\ldots$ & $\ldots$ & $8.24 \mathrm{E}+40$ & $\ldots$ & 0.14 \\
\hline 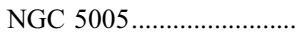 & 1996ai & -19.72 & 0.29 & Sbc & $1.29 \mathrm{E}+39$ & $\ldots$ & 0.16 \\
\hline 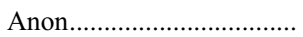 & 1996bl & $\ldots$ & $\ldots$ & $\mathrm{SBc}$ & $1.54 \mathrm{E}+40$ & 8.64 & 0.43 \\
\hline 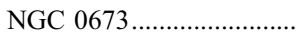 & 1996bo & -21.27 & 0.31 & $\mathrm{Sc}$ & $3.55 \mathrm{E}+40$ & 8.88 & 1.50 \\
\hline 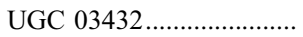 & 1996bv & -18.91 & 0.31 & Scd & $1.26 \mathrm{E}+40$ & 8.46 & 0.46 \\
\hline 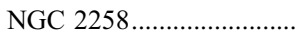 & $1997 \mathrm{E}$ & -20.31 & 0.29 & S0 & $\ldots$ & $\ldots$ & 0.01 \\
\hline 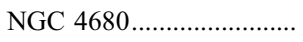 & $1997 b p$ & $\ldots$ & $\ldots$ & Pec & $1.21 \mathrm{E}+40$ & 8.96 & 1.11 \\
\hline 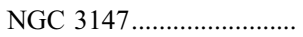 & $1997 b q$ & -21.59 & 0.28 & Sbc & $6.91 \mathrm{E}+39$ & $\ldots$ & 0.26 \\
\hline 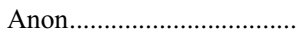 & $1997 \mathrm{br}$ & -17.68 & 0.32 & Sbd & $3.56 \mathrm{E}+39$ & 8.80 & 1.53 \\
\hline 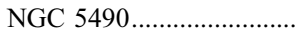 & $1997 \mathrm{cn}$ & -21.33 & 0.30 & $\mathrm{E}$ & $9.10 \mathrm{E}+37$ & $\ldots$ & 0.01 \\
\hline NGC $0105 \ldots \ldots \ldots \ldots \ldots \ldots \ldots$ & $1997 \mathrm{cw}$ & $\ldots$ & $\ldots$ & $\mathrm{Sab}$ & $2.07 \mathrm{E}+40$ & 8.79 & 1.23 \\
\hline 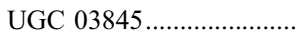 & 1997 do & -19.55 & 0.30 & $\mathrm{SBbc}$ & $8.51 \mathrm{E}+39$ & 8.62 & 0.92 \\
\hline 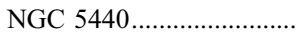 & 1998D & -20.35 & 0.31 & $\mathrm{Sa}$ & $1.36 \mathrm{E}+38$ & $\ldots$ & 0.04 \\
\hline 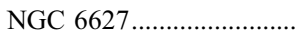 & 1998V & -20.5 & 0.39 & $\mathrm{Sb}$ & $3.46 \mathrm{E}+40$ & 9.01 & 0.74 \\
\hline 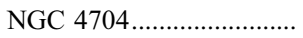 & $1998 \mathrm{ab}$ & -20.64 & 0.31 & Sbc pec & $2.07 \mathrm{E}+40$ & 9.09 & 0.54 \\
\hline 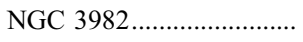 & 1998aq & -19.15 & 0.29 & $\mathrm{Sb}$ & $5.90 \mathrm{E}+39$ & 8.92 & 1.41 \\
\hline NGC $6495 \ldots \ldots \ldots \ldots \ldots \ldots \ldots$ & 1998bp & -20.02 & 0.30 & $\mathrm{E}$ & $1.75 \mathrm{E}+37$ & $\ldots$ & 0.01 \\
\hline 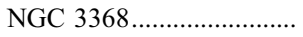 & 1998bu & $-20.21^{\mathrm{a}}$ & 0.22 & $\mathrm{Sab}$ & $5.28 \mathrm{E}+38$ & $\ldots$ & 0.06 \\
\hline 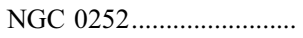 & 1998de & -20.84 & 0.28 & $\mathrm{Sab}$ & $1.05 \mathrm{E}+39$ & $\ldots$ & 0.04 \\
\hline CGCG 302-013 ................... & 1998di & $\ldots$ & $\ldots$ & $\ldots$ & $2.92 \mathrm{E}+38$ & $\ldots$ & 0.02 \\
\hline UGC $00139 \ldots \ldots \ldots \ldots \ldots \ldots$ & $1998 \mathrm{dk}$ & -19.43 & 0.35 & $\mathrm{Sc}$ & $1.10 \mathrm{E}+40$ & 8.85 & 0.92 \\
\hline UGCA $017 \ldots \ldots \ldots \ldots \ldots \ldots \ldots$ & $1998 \mathrm{dm}$ & $\ldots$ & $\ldots$ & $\mathrm{SBc}$ & $7.08 \mathrm{E}+39$ & 8.49 & 1.06 \\
\hline 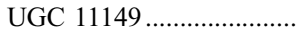 & $1998 d x$ & $\ldots$ & $\ldots$ & E2 & $9.66 \mathrm{E}+38$ & $\ldots$ & 0.01 \\
\hline 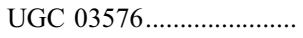 & $1998 \mathrm{ec}$ & -20.22 & 0.31 & $\mathrm{SBb}$ & $6.18 \mathrm{E}+39$ & $\ldots$ & 0.18 \\
\hline 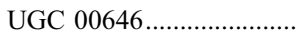 & 1998ef & -19.48 & 0.30 & $\mathrm{Sb}$ & $0.00 \mathrm{E}+00$ & $\ldots$ & 0.02 \\
\hline 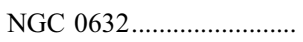 & $1998 \mathrm{es}$ & -19.94 & 0.28 & $S 0^{d}$ & $1.20 \mathrm{E}+40$ & 8.96 & 1.66 \\
\hline CGCG 180-022 ................. & 1999X & $\ldots$ & $\ldots$ & $\ldots$ & $4.79 \mathrm{E}+38$ & $\ldots$ & 0.03 \\
\hline 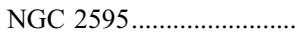 & 1999aа & -20.98 & 0.38 & $\mathrm{Sc}$ & $1.21 \mathrm{E}+40$ & $\ldots$ & 0.52 \\
\hline 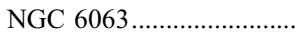 & $1999 \mathrm{ac}$ & -19.22 & 0.30 & Scd & $3.51 \mathrm{E}+39$ & 8.90 & 0.44 \\
\hline 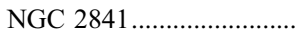 & 1999by & -19.66 & 0.28 & $\mathrm{Sb}$ & $1.76 \mathrm{E}+38$ & $\ldots$ & 0.04 \\
\hline 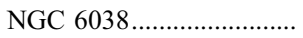 & $1999 \mathrm{cc}$ & -21.31 & 0.30 & $\mathrm{Sc}$ & $3.12 \mathrm{E}+40$ & $\ldots$ & 0.37 \\
\hline 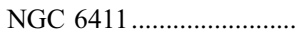 & 1999da & -20.67 & 0.28 & $\mathrm{E}$ & $0.00 \mathrm{E}+00$ & $\ldots$ & 0.01 \\
\hline 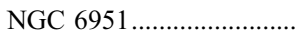 & $2000 \mathrm{E}$ & -19.57 & 0.29 & $\mathrm{Sbc}$ & $1.24 \mathrm{E}+39$ & $\ldots$ & 0.15 \\
\hline
\end{tabular}

\footnotetext{
${ }^{a}$ Gibson et al. (2000).

b Interacting galaxy.

c Hamuy et al. (1996a).

d Starburst core.
} 
TABLE 5

Type Ia Supernova Characterization

\begin{tabular}{|c|c|c|c|c|}
\hline $\begin{array}{c}\text { Galaxy } \\
\text { (1) }\end{array}$ & $\begin{array}{l}\mathrm{SN} \\
(2)\end{array}$ & $\begin{array}{c}\Delta m_{15}(B) \\
(\mathrm{mag}) \\
(3)\end{array}$ & $\begin{array}{c}\sigma \\
(\mathrm{mag}) \\
(4)\end{array}$ & $\begin{array}{l}\text { Hubble Residual } \\
\text { (mag) } \\
\text { (5) }\end{array}$ \\
\hline NGC $4536 \ldots \ldots \ldots \ldots . . . .$. & 1981B & 1.10 & 0.07 & $\ldots$ \\
\hline NGC $3627 \ldots \ldots \ldots \ldots . . . . .$. & 1989B & 1.31 & 0.07 & $\ldots$ \\
\hline NGC $4639 . . . \ldots \ldots \ldots . . . .$. & $1990 \mathrm{~N}$ & 1.07 & 0.05 & $\ldots$ \\
\hline CGCG $111016 \ldots \ldots$. & 19900 & 0.96 & 0.10 & 0.11 \\
\hline NGC $4527 \ldots \ldots \ldots \ldots . . . . .$. & $1991 \mathrm{~T}$ & 0.94 & 0.05 & $\ldots$ \\
\hline IC $4232 \ldots \ldots \ldots \ldots \ldots . . . . . . . .$. & $1991 \mathrm{U}$ & 1.06 & 0.10 & -0.31 \\
\hline NGC $4374 .$. & $1991 b g$ & 1.93 & 0.10 & $\ldots$ \\
\hline Anon....... & $1992 J$ & 1.56 & 0.10 & -0.23 \\
\hline IC $3690 \ldots \ldots \ldots \ldots \ldots \ldots$ & 1992P & 0.87 & 0.10 & 0.20 \\
\hline Anon............................ & $1992 \mathrm{ag}$ & 1.19 & 0.10 & -0.13 \\
\hline 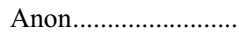 & $1992 b p$ & 1.32 & 0.10 & -0.12 \\
\hline CGCG $307023 \ldots \ldots$. & $1993 \mathrm{ac}$ & 1.19 & 0.10 & 0.06 \\
\hline NGC $4526 \ldots \ldots \ldots \ldots . . . .$. & 1994D & 1.32 & 0.05 & $\ldots$ \\
\hline NGC $4493 \ldots \ldots \ldots \ldots . . . . . .$. & $1994 \mathrm{M}$ & 1.44 & 0.10 & -0.19 \\
\hline CGCG 224-104....... & 1994Q & 1.03 & 0.10 & -0.12 \\
\hline NGC $4495 \ldots \ldots \ldots \ldots . . . .$. & $1994 \mathrm{~S}$ & 1.10 & 0.10 & 0.03 \\
\hline NGC $3370 \ldots \ldots \ldots . . . . . .$. & 1994ae & 0.86 & 0.05 & $\ldots$ \\
\hline NGC $2962 \ldots \ldots \ldots \ldots \ldots$ & 1995D & 0.99 & 0.05 & $\ldots$ \\
\hline NGC $2441 \ldots \ldots \ldots \ldots . . . . .$. & $1995 \mathrm{E}$ & 1.06 & 0.05 & $\ldots$ \\
\hline 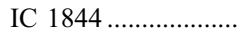 & 1995ak & 1.26 & 0.10 & -0.34 \\
\hline NGC $3021 \ldots \ldots \ldots \ldots . . .$. & 1995al & 0.83 & 0.05 & $\ldots$ \\
\hline UGC $03151 \ldots \ldots \ldots \ldots$. & $1995 b d$ & 0.84 & 0.05 & -0.13 \\
\hline Anon............................. & $1996 \mathrm{C}$ & 0.97 & 0.10 & 0.32 \\
\hline NGC $2935 \ldots \ldots \ldots \ldots . . . .$. & $1996 Z$ & 1.22 & 0.10 & $\ldots$ \\
\hline Anon............................ & 1996ab & 0.87 & $\ldots$ & 0.23 \\
\hline NGC $5005 \ldots \ldots \ldots \ldots \ldots$ & 1996ai & 0.99 & 0.10 & $\ldots$ \\
\hline 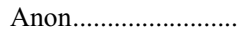 & 1996bl & 1.17 & 0.10 & 0.12 \\
\hline NGC $0673 \ldots \ldots \ldots \ldots . . . . .$. & 1996bo & 1.25 & 0.05 & $\ldots$ \\
\hline UGC $03432 \ldots \ldots \ldots \ldots$ & 1996bv & 0.93 & 0.10 & -0.15 \\
\hline NGC $2258 \ldots \ldots \ldots \ldots . . . .$. & 1997E & 1.39 & 0.06 & 0.14 \\
\hline NGC $4680 \ldots \ldots \ldots \ldots . . . .$. & 1997bp & 1.00 & 0.05 & -0.16 \\
\hline NGC $3147 \ldots \ldots \ldots \ldots . . . . .$. & $1997 \mathrm{bq}$ & 1.00 & 0.05 & -0.08 \\
\hline 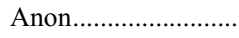 & $1997 b r$ & 1.02 & 0.06 & $\ldots$ \\
\hline NGC $5490 \ldots \ldots \ldots \ldots . . .$. & $1997 \mathrm{cn}$ & 1.90 & 0.05 & 0.16 \\
\hline NGC $0105 \ldots \ldots \ldots . . . . .$. & $1997 \mathrm{cw}$ & 1.02 & 0.10 & -0.07 \\
\hline UGC $03845 \ldots \ldots \ldots \ldots$ & 1997do & 0.99 & 0.10 & 0.24 \\
\hline NGC $5440 \ldots \ldots \ldots \ldots . . . .$. & 1998D & $\ldots$ & $\ldots$ & 0.02 \\
\hline NGC $6627 \ldots \ldots \ldots \ldots . . . .$. & 1998V & 1.06 & 0.05 & -0.03 \\
\hline NGC $4704 \ldots \ldots \ldots \ldots \ldots$ & $1998 \mathrm{ab}$ & 0.88 & 0.17 & -0.12 \\
\hline NGC $3982 \ldots \ldots \ldots \ldots . . . .$. & $1998 \mathrm{aq}$ & 1.14 & $\ldots$ & $\ldots$ \\
\hline NGC $6495 \ldots \ldots \ldots \ldots . . . .$. & 1998bp & 1.83 & 0.06 & -0.05 \\
\hline NGC $3368 \ldots \ldots \ldots \ldots . . .$. & 1998bu & 1.01 & 0.05 & $\ldots$ \\
\hline NGC $0252 \ldots \ldots \ldots \ldots . . .$. & 1998de & 1.93 & 0.05 & 0.02 \\
\hline CGCG $302013 \ldots \ldots$. & 1998di & $\ldots$ & $\ldots$ & $\ldots$ \\
\hline UGC $00139 \ldots \ldots \ldots \ldots$ & $1998 \mathrm{dk}$ & 1.05 & 0.10 & 0.06 \\
\hline UGCA $017 \ldots \ldots \ldots \ldots$ & $1998 \mathrm{dm}$ & 1.07 & 0.06 & $\ldots$ \\
\hline UGC $11149 \ldots \ldots \ldots \ldots$ & $1998 \mathrm{dx}$ & 1.55 & 0.09 & -0.08 \\
\hline UGC $03576 \ldots \ldots \ldots \ldots . . .$. & $1998 \mathrm{ec}$ & 1.08 & 0.09 & 0.09 \\
\hline UGC $00646 \ldots \ldots \ldots \ldots . . .$. & $1998 \mathrm{ef}$ & 0.97 & 0.10 & $\ldots$ \\
\hline NGC $0632 \ldots \ldots \ldots \ldots . . .$. & 1998 es & 0.87 & 0.08 & -0.06 \\
\hline CGCG $180022 \ldots \ldots$. & 1999X & 1.11 & 0.08 & 0.04 \\
\hline NGC $2595 \ldots \ldots \ldots \ldots . . .$. & 1999aа & 0.85 & 0.08 & $\ldots$ \\
\hline NGC $6063 \ldots \ldots \ldots \ldots . . . .$. & 1999ac & 1.00 & 0.08 & 0.17 \\
\hline NGC $2841 \ldots \ldots \ldots \ldots . . . .$. & 1999by & 1.87 & $\ldots$ & $\ldots$ \\
\hline NGC $6038 \ldots \ldots \ldots \ldots . . . . .$. & $1999 \mathrm{cc}$ & 1.46 & 0.05 & 0.05 \\
\hline NGC $6411 \ldots \ldots \ldots \ldots . . . . .$. & 1999da & 1.94 & $\cdots$ & 0.10 \\
\hline NGC $6951 \ldots \ldots \ldots \ldots . . . .$. & $2000 \mathrm{E}$ & 0.94 & 0.50 & $\ldots$ \\
\hline
\end{tabular}

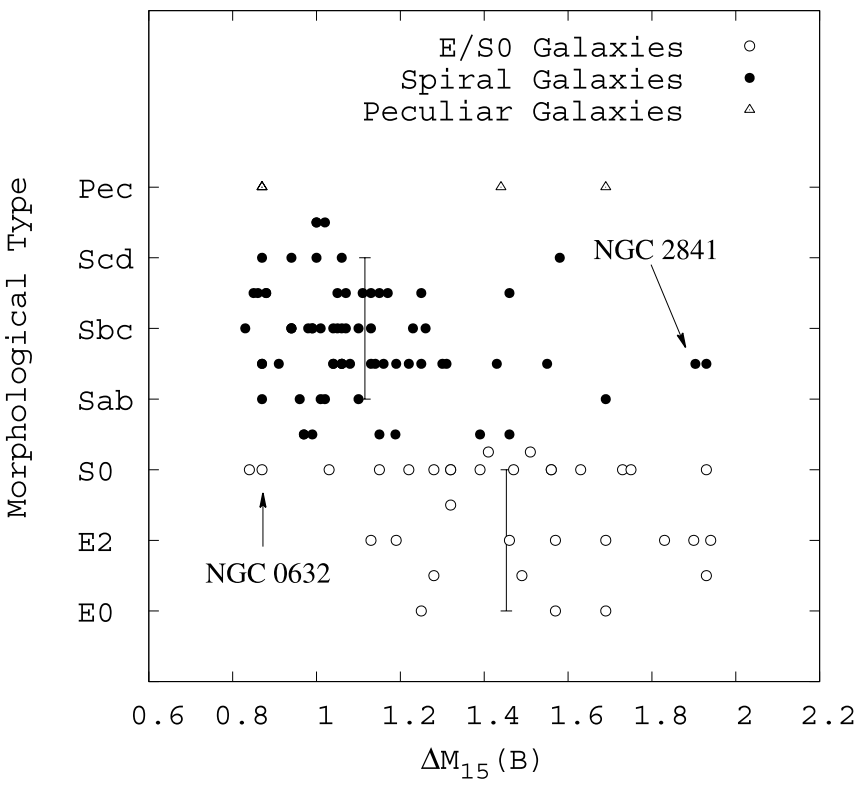

FIG. 1.-Morphological type vs. decline rate of SNe. Highlighted are NGC 2841, a categorized Sa galaxy with spectral features of an elliptical, and NGC 0632, a categorized S0 with the strong emission typically seen in late-type galaxies. Vertical markers highlight the average decline rates for both early- and late-type host galaxies.

helpful, though incomplete picture of the host properties and that a more detailed host galaxy characterization is necessary.

\subsubsection{Metallicity}

Theoretical studies conducted by Höflich et al. (1998), Umeda et al. (1999), and Höflich et al. (2000, hereafter HNUW00), along with analytical analysis by TBT03, have suggested that the initial metallicity of the SN Ia progenitor can have a small, but possibly significant effect (particularly at high metallicity) on the luminosity of the resultant supernova. HNUW00 pointed out that the metallicity of the progenitor on the main sequence can affect the mass of the interior $\mathrm{C} / \mathrm{O}$ core left behind as a WD and ultimately affect the amount of ${ }^{56} \mathrm{Ni}$ produced in the explosion and the peak luminosity of the SN Ia. TBT03 analytically demonstrated that a factor of 3 variation in progenitor metallicity results in an $\sim 25 \%$ variation is the mass of ${ }^{56} \mathrm{Ni}$ ejected during the SN Ia event. If one allows for the sedimentation of ${ }^{22} \mathrm{Ne}$, then the variation can be as high as $50 \%$. Furthermore, Umeda et al. (1999) suggested that the carbon mass fraction in a CO white dwarf is dependent on the metallicity of the environment in which it was formed. They further proposed that the observed diversity in SNe Ia brightness is a consequence of this phenomenon, with the smaller progenitor carbon fractions leading to dimmer supernova. Under the assumption that higher galactic metallicity is proportional to the average progenitor metallicity, we set out to investigate these theoretical results through observation.

Figure 2 shows the relationship between host galaxy metallicity and SNe Ia decline rate for our sample, with a distinction drawn between elliptical and spiral galaxies. Two ellipticals and one spiral galaxy have been included with metallicities given in H00. A regression line is fitted to our host sample of spiral galaxies, and we find a small correlation suggesting that higher metallicity spiral host galaxies produce faster declining, less luminous SNe. However, a Monte Carlo simulation places this correlation at only the $70 \%$ confidence level. The simulation involved generating 25 evenly distributed metallicities between 8.3 and 9.4, assigning each one a $\Delta m_{15}(B)$ from our data and 


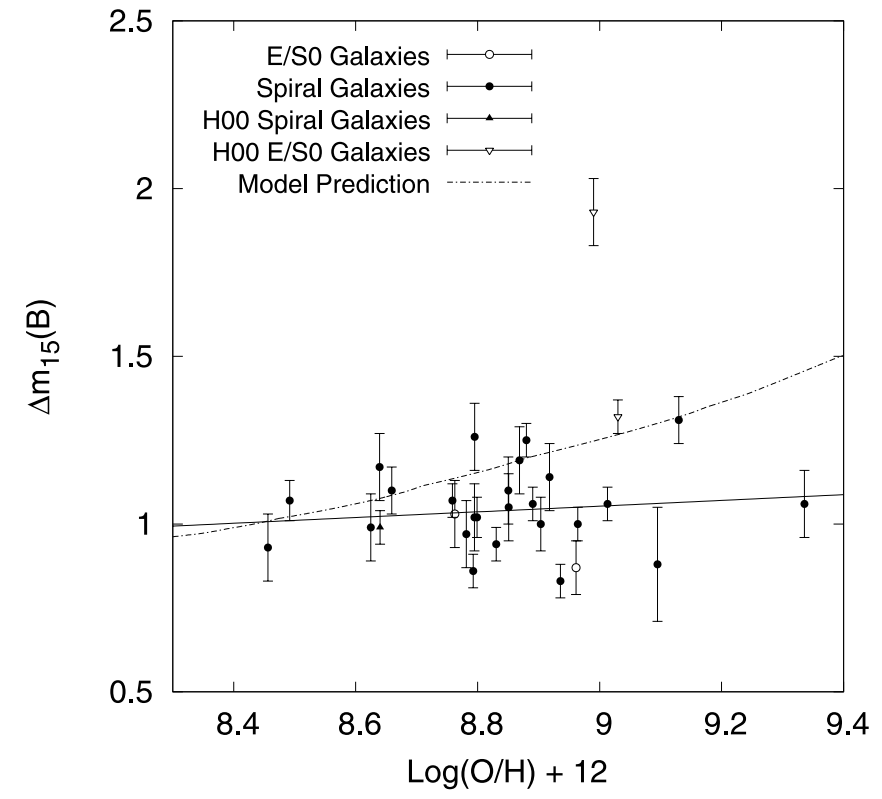

FIG. 2.-Decline rate dependence on metallicity. SN Ia samples of late types show a tendency for high-metallicity galaxies to host fainter $\mathrm{SNe} \mathrm{Ia}$, at the $70 \%$ confidence level. The addition of $\mathrm{H} 00$ galaxies further suggests a decrease in SN brightness with increased metallicity from early- to late-type galaxies. The solid line is a linear best fit to the data, while the broken line represents the predicted trend governed by the studies of TBT03, Höflich et al. (2002), and Garnavich et al. (2004).

determining after a number of trials the probability of obtaining a best-fit slope greater than or equal to the absolute value of the slope seen in Figure 2. This does not suggest that metallicity has a great affect on the luminosity of Type Ia supernovae.

A comparison between the late-type galaxies of $\mathrm{H} 00$ and our spiral galaxies at the same metallicity shows a wide dispersion in decline rate at a fixed metallicity, likewise suggesting a weak dependence of SN Ia decline rate on the environment metallicity. However, the presence of two metal-rich ellipticals from $\mathrm{H} 00$ that hosted SNe Ia with a higher decline rate on average than our spirals could hint at an overall increase in $\Delta m_{15}(B)$ with metallicity across the full Hubble sequence. Such a trend would support the predictions made by the analytical models of TBT03. We use the DD numerical models of Höflich et al. (2002) and the empirical relations of Garnavich et al. (2004) to convert the predictions of TBT03 to the observed parameter. The predicted relation is shown in Figure 2 (dot-dashed line). Oxygen abundances were converted into iron abundances using the $[\mathrm{O} / \mathrm{Fe}]$ to $[\mathrm{Fe} / \mathrm{H}]$ relation predicted by the three-component mixing models of Qian \& Wasserburg (2001). The ${ }^{56} \mathrm{Ni}$ masses were calculated for the iron abundances according to the analytical model of TBT03. The decline rate lower limit in the plotted range is set by the $M_{\mathrm{Ni}}$ versus metallicity relation presented in TBT03. We assume a fiducial SN Ia $M_{\mathrm{Ni}}$ production of approximately $0.64 M_{\odot}$. Varying this fiducial mass acts to vary the lowmetallicity decline rate limit. Interpolation of the $M_{V}$ versus $M_{\mathrm{Ni}}$ plot in Höflich et al. (2002) yielded the corresponding $M_{V}$ for each ${ }^{56} \mathrm{Ni}$ mass. Finally, we found corresponding decline rates through the empirical $M_{V}$ versus $\Delta m_{15}(B)$ relation presented in Garnavich et al. (2004). This predicted curve is not meant to provide for a detailed comparison with the observations but rather to convey the general metallicity decline rate trend predicted by TBT03. The curve implies a minimal dependence of Type Ia SN luminosity on metallicity for metallicities below solar. However, as progenitor metallicity increases well above solar, the predicted dependence becomes steeper, resulting in significantly fainter $\mathrm{SNe} \mathrm{Ia}$.

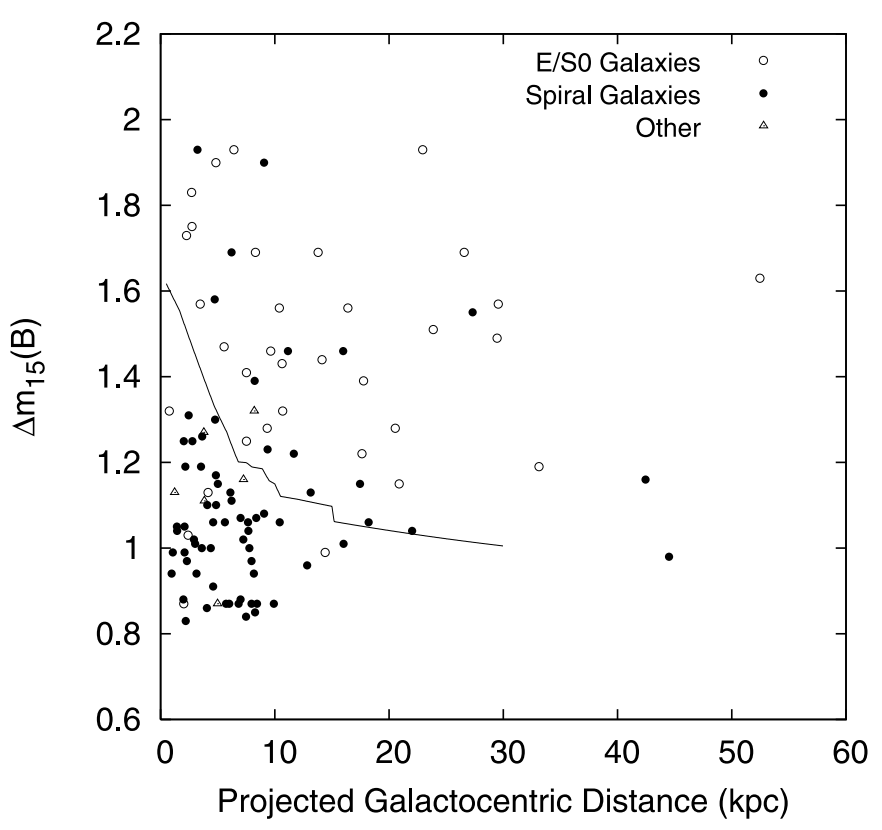

FIG. 3.-Type Ia SNe decline rate vs. projected galactocentric distance. The solid line represents the predicted decline in $\Delta m_{15}(B)$ with PGD due to a decrease in metallicity with increasing PGD (Andrievsky et al. 2004).

Figure 3 shows the projected galactocentric distances (PGDs) of Type Ia supernovae versus $\Delta m_{15}(B)$. The SNe were compiled from the list presented in Phillips et al. (1999), Jha (2002), Riess et al. (1999), and Krisciunas et al. (2004). Projected offsets were obtained from the Harvard CfA List of Supernovae ${ }^{3}$ and the Central Bureau for Astronomical Telegrams (CBAT). ${ }^{4}$ Hubble flow luminosity distances were calculated from the SN redshift, assuming cosmological parameters $H_{0}=72 \mathrm{~km} \mathrm{~s}^{-1} \mathrm{Mpc}^{-1}$, $\Omega_{M}=0.28$, and $\Omega_{\Lambda}=0.72$, while non-Hubble flow distances were estimated using the $\mathrm{SNe}$ luminosities. A more even distribution in decline rates is observed for supernovae hosted by elliptical galaxies than those hosted by their smaller spiral counterparts. The relevance of this plot to metallicity becomes clear when we compare it to the expected metallicity gradient across a typical spiral galaxy. Recent results by Andrievsky et al. (2004) have indicated that there is a drop of $0.6 \mathrm{dex}$ in $[\mathrm{Fe} / \mathrm{H}]$ across the disk of the Milky Way from approximately 4.0 to $16 \mathrm{kpc}$. Assuming the Milky Way to be adequately representative of a typical spiral galaxy, we can find a theoretical relation between the SN PGD and its decline rate using the methods detailed above. The relation is plotted in Figure 3, and it suggests that among SNe hosted by spiral galaxies, the fainter events are predicted to reside nearest the galactic center-a prediction that stands in contrast to the observations in Figure 3 showing the brighter events clustering at low PGDs.

We also compared the SN hosts with two larger sets of galaxies in the hope of shedding light on possible systematic selection effects or, more interestingly, on evolutionary effects present in the discovery of SNe Ia. The first set was the NFGS sample. The NFGS is a collection of integrated and nuclear spectroscopy for approximately 200 galaxies in the near field. The sample was analyzed with the FAST spectrograph operating on the Tillinghast telescope and includes galaxies of every morphological type covering $8 \mathrm{mag}$ in luminosity (Jansen et al. 2000, hereafter J00).

\footnotetext{
3 At http://cfa-www.harvard.edu/cfa/ps/lists/Supernovae.html.

4 At http://cfa-www.harvard.edu/iau/cbat.html.
} 


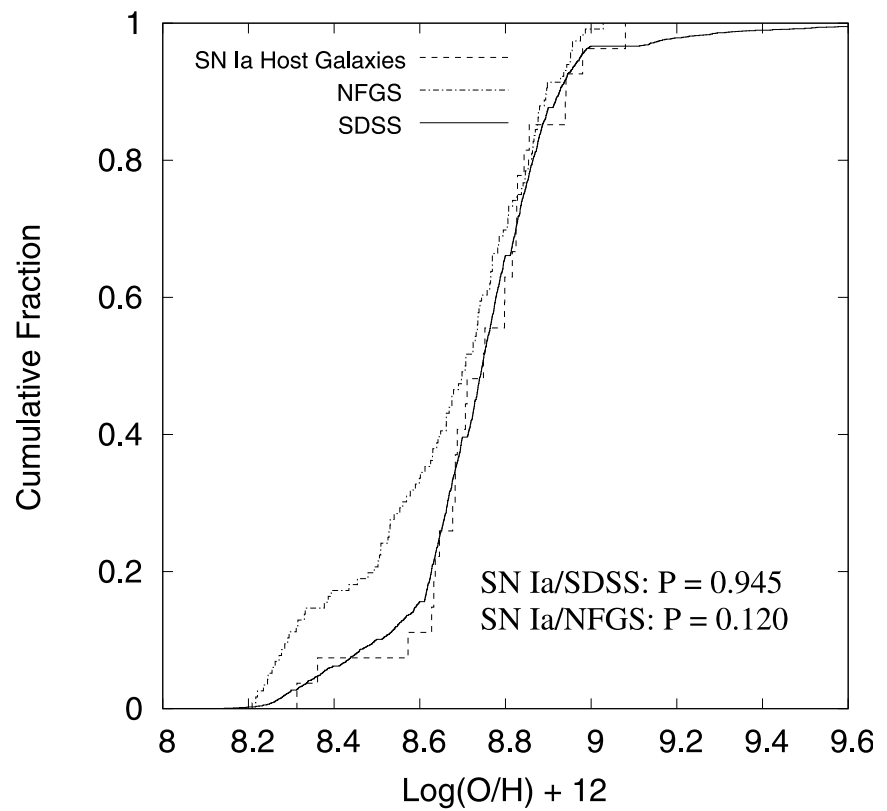

FIG. 4.-Cumulative fraction plots for our SN host galaxy sample, NFGS galaxy sample, and the SDSS galaxy sample. A K-S test finds the probability of the host galaxy distribution and the NFGS distribution being drawn from the same distribution to be $12.0 \%$. Moreover, we find that the likelihood of the host galaxy sample and the SDSS sample being drawn from identical galaxy distributions to be $94.5 \%$.
We were able to calculate metallicities for 116 galaxies using the integrated emission-line EW presented in J00. The second comparison distribution is a set of approximately 9000 SDSS galaxies whose spectroscopic line strength data were obtained through the Carnegie Mellon University-University of Pittsburgh's SDSS Value-Added Catalog (CMU-VAC). We were able to obtain 3133 galaxy metallicities from the SDSS sample using the EW line strengths obtained from the CMU-VAC. For consistency, host galaxy metallicities used for comparison with the NFGS and the SDSS metallicity distributions were calculated from emission-line EW ratios, and both the NFGS and SDSS samples were limited to emission-line galaxies.

We performed a Kolmogorov-Smirnov test to determine the likelihood that our host galaxies were drawn from similar distributions as the NFGS sample and the SDSS sample. Figures 4 and 5 show the cumulative fraction plots (CFPs) and the histograms for the metallicity distributions in each sample, respectively. We find from a K-S test that the observed SN host galaxies could be drawn from the NFGS sample (12.0\% probability) or from the SDSS sample (94.5\%). The consistency between SN Ia host distribution, the NFGS distribution, and particularly the SDSS distributions implies a high probability that the SN hosts do not have a unique metallicity signature as compared to a general sample offield galaxies, suggesting that the probability of an SN Ia occurring is not strongly dependent on the metallicity of the host galaxy in the range $0.05 Z_{\odot} \lesssim Z \gtrsim 3.5 Z_{\odot}$.

\subsubsection{Age}

In each of Figures $2-5$ the observed behaviors fall into one of two categories. They either show a negligible effect of
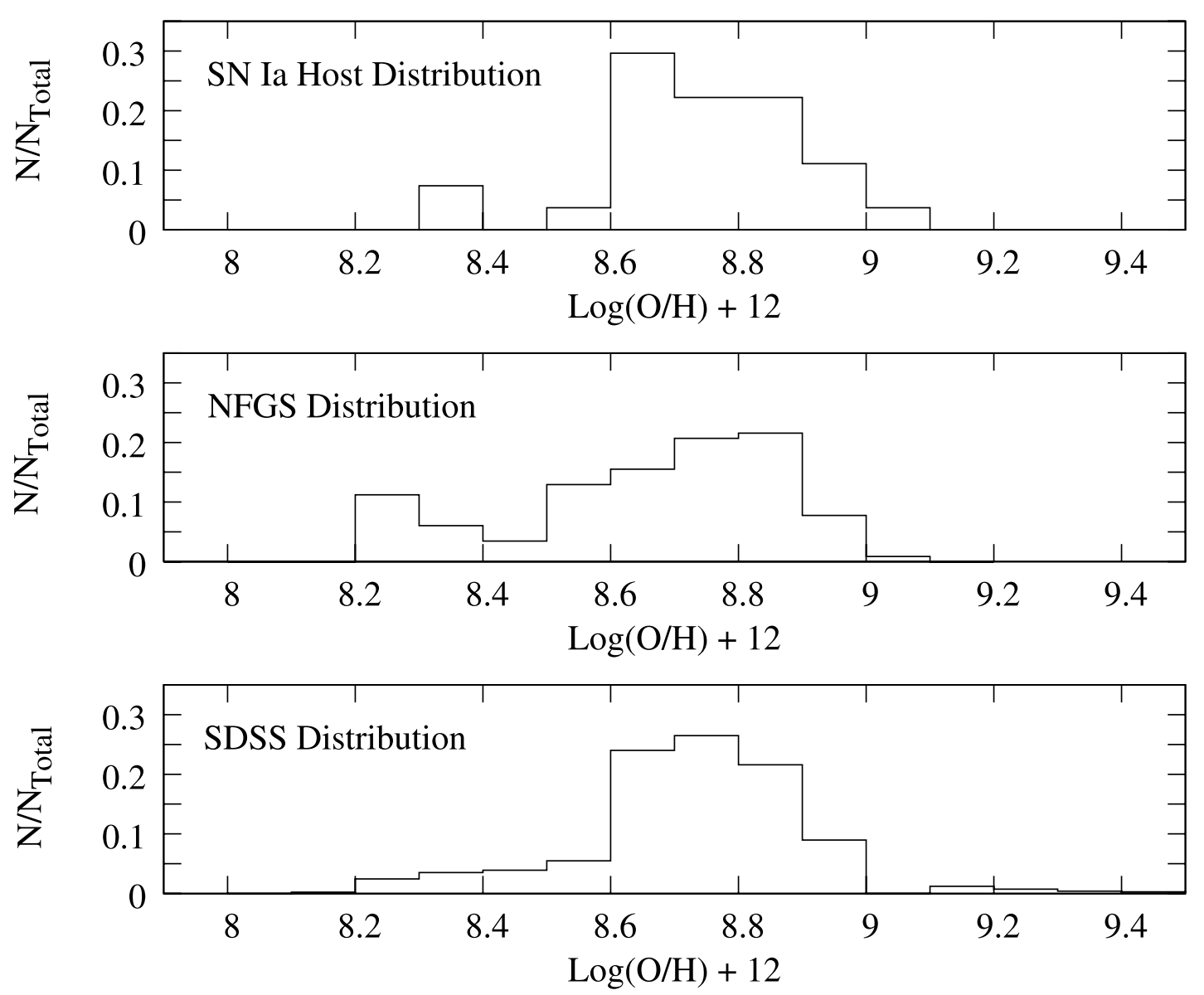

FIG. 5.-Metallicity distributions for the SN host galaxy, NFGS, and SDSS samples. 

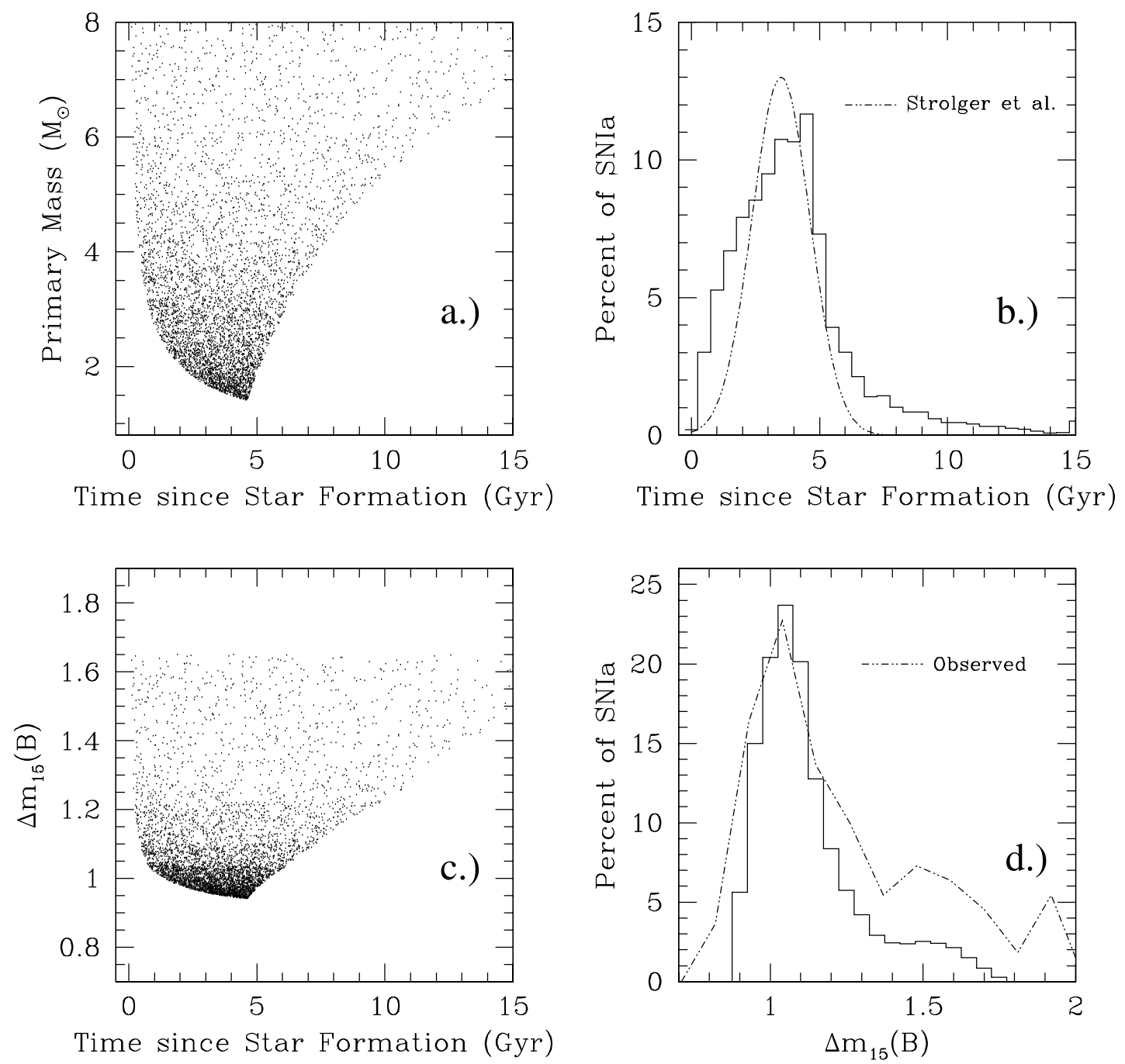

FIG. 6.-Effect of progenitor age on the variations in Type Ia SNe luminosity. Progenitor age as a function of primary mass is given in (a) assuming an IMF as published in Kroupa et al. (1993). The distribution of progenitor age with the predictions of Strolger et al. (2004) overplotted (b). The SN Ia decline rate as a function of progenitor age $(c)$, and the distribution of decline rates that results $(d)$.

metallicity on the luminosity of Type Ia supernovae or they show a trend that opposes current theoretical predictions. In either case, the results suggest that metallicity is unlikely to be the primary contributor to the variability observed in the peak luminosities of Type Ia supernovae. Another property known to be correlated with host galaxy morphology is population age. The population age, which we use as an approximation of the progenitor age, may be able to explain the luminosity variations of $\mathrm{SNe}$ Ia and the correlation of these luminosities with host galaxy morphological type (Fig. 1). The progenitor age is the amount of time between the birth of the progenitor and the time of the supernova event. We can investigate the effect of progenitor age on the SN Ia luminosity distribution using a simple model inspired by Umeda et al. (1999). This is shown in Figure 6. We first adopted the single degenerate Chandrasekhar mass model for our SN progenitor system. We then randomly selected pairs of stars, $M_{1}$ and $M_{2}$, from a distribution of stars consistent with the IMF in Kroupa et al. (1993). A constraint is placed on the secondary mass, requiring

$$
\left(M_{2}-M_{\mathrm{WD}_{2}}\right)+M_{\mathrm{WD}_{1}} \geq 1.4 M_{\odot},
$$

where $M_{\mathrm{WD}_{1}}$ and $M_{\mathrm{WD}_{2}}$ are the subsequent WD masses corresponding to main-sequence masses $M_{1}$ and $M_{2}$ (Dominguez et al. 1999). This ensured that the secondary possessed the necessary mass required for $M_{\mathrm{WD}_{1}}$ to attain the Chandrasekhar limit through mass accretion. To prevent the inclusion of stars that explode as core collapse $\mathrm{SNe}$, we limited $M_{1}$ and $M_{2}$ to be $\leq 8 M_{\odot}$. Finally, we assumed that the $\mathrm{SNe}$ explosion occurs soon after the main-sequence lifetime of the secondary, thus setting the progenitor age, i.e., the delay time, by the lifetime of the secondary.

Figure $6 a$ shows the distribution of progenitor age as a function of the $M_{1}$. For a given primary mass, there is an upper and lower limit placed on the mass of the secondary and thus on the lifetime of the secondary. The lower limit on the progenitor mass arises from the requirement that the secondary star have enough mass to put the $M_{\mathrm{WD}_{1}}$ over the Chandrasekhar limit. On the other hand, the upper limit arises from a need for the secondary to have a lower mass than the primary. Allowing the secondary to have a greater mass would simply exchange the respective labels of primary and secondary. Given the nature of stellar evolution, an upper limit on mass becomes a lower limit on age and vice versa. The point where the two limits meet represents a system in which $M_{1}=M_{2}$. Binning the age axis in Figure $6 a$ yields the age distribution of SNe Ia in Figure $6 b$. We have overplotted the derived distribution published in Strolger et al. (2004) for the 


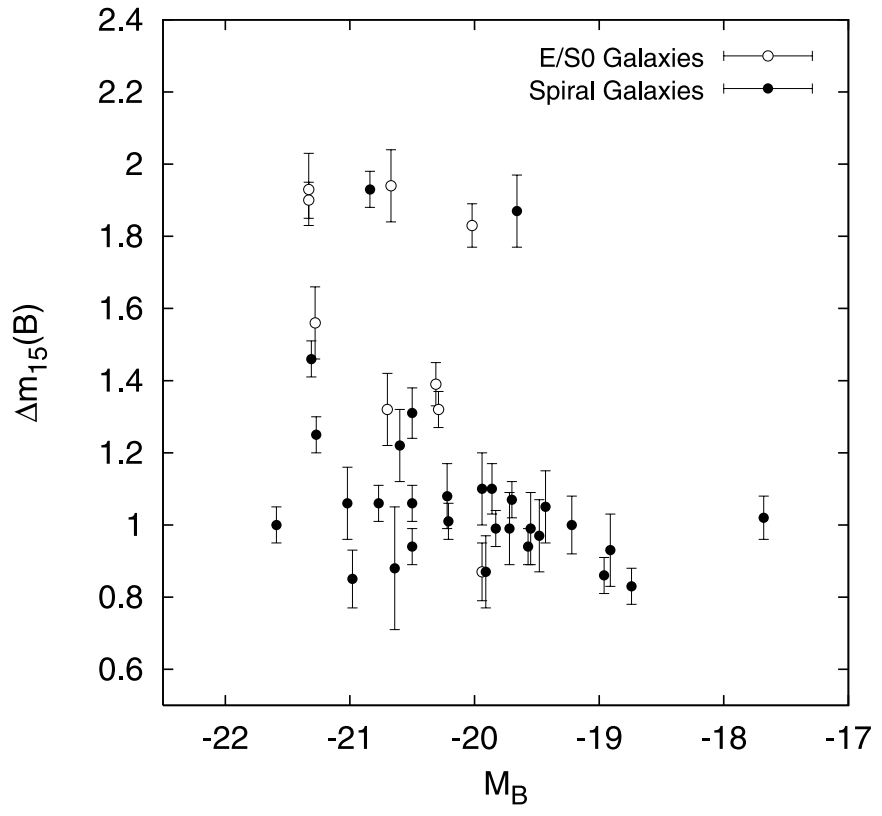

FIG. 7.-Decline rate vs. host galaxy absolute magnitude. The distribution shows an absence of dimmer SNe Ia in the low-luminosity host galaxy regime.

Type Ia supernovae delay time, or the time between progenitor formation and the SN event. Our simple model is in good agreement with an average Type Ia supernova delay time of around 3 Gyr.

Next, we set out to determine the expected effects that the progenitor age has on Type Ia supernova decline rate by first approximating a linear fit to the plot of core mass versus mainsequence mass from Dominguez et al. (1999) and converting our primary masses into WD masses. Umeda et al. (1999) postulated that the $M_{56 \mathrm{Ni}}$, and consequently the brightness of the SN Ia, increases as the $\mathrm{C} / \mathrm{O}$ ratio of the progenitor increases. Based on the postulate, they developed a model describing this dependency. Although the model should be treated with caution, given that it is based on an unproven postulate, we use it in our toy model merely to provide a rough understanding on the effects of age on the decline rates of SNe Ia. Therefore, using these ${ }^{56} \mathrm{Ni}$ yields and the $M_{56 \mathrm{Ni}}$ to $\Delta m_{15}(B)$ relations described in $\S 3.2 .1$, we converted the WD masses to the expected SN decline rates. The resultant decline rate versus age scatter plot is shown in Figure $6 c$. By binning the $\Delta m_{15}(B)$ axis we obtain the expected decline rate distribution for Type Ia supernovae in Figure $6 d$. This figure shows that the age of the progenitor can result in a variation in decline rate similar to that which is observed for SNe Ia. This consistency is compelling evidence for age, not metallicity, acting as the primary source of SN Ia diversity. Future studies will obtain spectra of elliptical galaxies for use in absorption-line metallicity and age estimations using the stellar population synthesis models of Worthey (1994). This will enable us to investigate the effects of age on the properties of Type Ia supernovae directly.

\subsubsection{Absolute B Magnitude}

H00 showed, for a sample of nearby SN Ia host galaxies, a trend (with high dispersion) indicating that the SN Ia decline rate increases, and consequently its maximum luminosity decreases, with increased host galaxy luminosity. Henry \& Worthey (1999) were able to show that the integrated luminosity of a galaxy is correlated with its global metallicity, as the brighter, more massive galaxies were able to better retain $\mathrm{SN}$ heavy-metal ejecta.
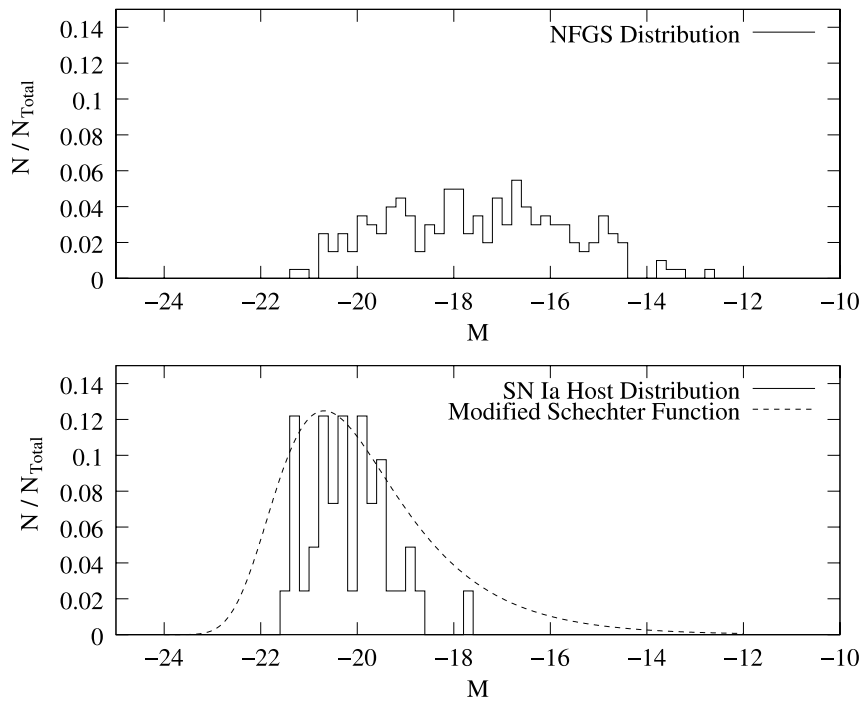

FIG. 8.-Absolute magnitude distribution for the NFGS galaxy and SN Ia host galaxy samples. The histogram reveals the host galaxy distribution to be statistically brighter than the NFGS galaxy sample. The broken line is the theoretical SNe Ia distribution as predicted by our MSF.

Therefore, H00 argued that any correlation observed between decline rate and absolute magnitude might manifest itself as a correlation between decline rate and metallicity. Figure 7 shows the absolute $B$ magnitude of our host galaxy sample versus $\Delta m_{15}(B)$. Although Figure 7 does not show the gradual trend observed by $\mathrm{H} 00$, the plot does show less scatter for the least luminous galaxies, likely due to a combination of two effects. The first is a selection effect brought about by fewer SNe occurring in smaller galaxies. Such an effect would suggest that if we had more $\mathrm{SNe}$ from low-luminosity galaxies, then the bias toward bright $\mathrm{SNe}$ Ia in the low-luminosity galaxy regime would disappear. However, according to Figure 1, fainter SNe are predominantly hosted by elliptical galaxies, which are on average brighter than spiral galaxies. Therefore, we see in Figure 7 a tendency for fainter SNe to be hosted by large, bright galaxies. The combination of these two effects contributes to the distribution observed in Figure 7.

Figure 8 shows the histograms of the NFGS and the SN Ia host galaxy distributions. The two distributions are clearly dissimilar owing to the nearly $2.5 \mathrm{mag}$ discrepancy observed between the average magnitudes of the respective galaxy samples. The NFGSselected galaxies cover a wide range of luminosities ( $8 \mathrm{mag}$ ), and thus we would expect a broad distribution with little evidence of bias, as seen in Figure 8. On the other hand, the SN Ia host galaxy selection process was not subject to such regulations. However, several selection effects were ultimately present in the SN Ia search. First, the selection of target galaxies in the supernova Ia search often involved point searches that focused on well-known and inevitably more luminous targets that would bias the SN host galaxies toward the higher luminosity regime. Furthermore, bias was introduced due to the nonuniformity of the luminosity function (LF) of galaxies. The form of the LF known as the Schechter function (Schechter 1976),

$$
\Phi(M) \sim 10^{-0.4(\alpha+1) M} e^{-10^{0.4\left(M^{*}-M\right)},}
$$

with $\alpha=-1.17$ and $M^{*}=-20.73$ given by the Century Survey (Geller et al. 1997), is plotted in Figure 9.

The LF illustrates that high-luminosity galaxies are in the minority throughout the universe. Consequently, the probability of finding a SN Ia in a high-luminosity galaxy is small, and the 


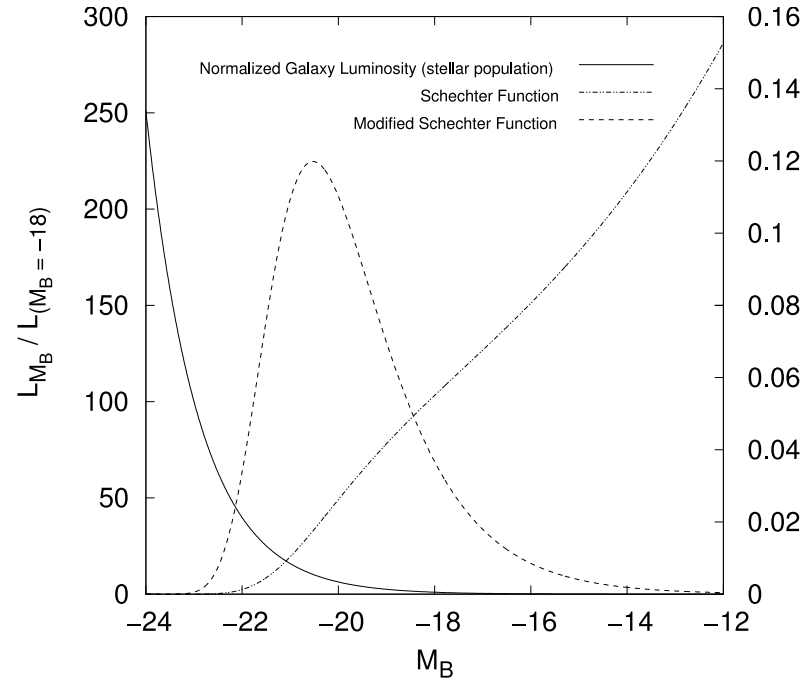

FIG. 9.-MSF. The solid line shows the relative galactic luminosity and consequently the galactic stellar population as a function of galaxy absolute $B$ magnitude. The dot-dot-dashed line shows the Schechter function as described by Schechter (1976). The MSF (dashed line) gives the relative probability of finding a Type Ia $\mathrm{SN}$ as a function of galactic $B$-filter magnitude.

overall $M_{B}$ distribution of SN Ia host galaxies will be shifted to the lower magnitudes. However, higher luminosity galaxies inherently have larger populations of stars than their lower luminosity counterparts. Figure 9 further reflects how the relative luminosity and the stellar population of a galaxy changes with absolute $B$ magnitude [function normalized to $L_{\left(M_{B}=-18\right)}=1$ ]. This curve assumes the luminosity in the $B$ band to be an adequate tracer of galactic mass. Although not the best tracer (Mannucci et al. 2005), in the absence of good near-infrared $H$ - or $K$-band measurements, the $B$ band should be sufficient for this analysis. We can see from the figure that although highluminosity galaxies are more rare than low-luminosity galaxies, they possess more stars and thus have an increased probability of hosting a SN Ia. We can investigate the combined effects of these

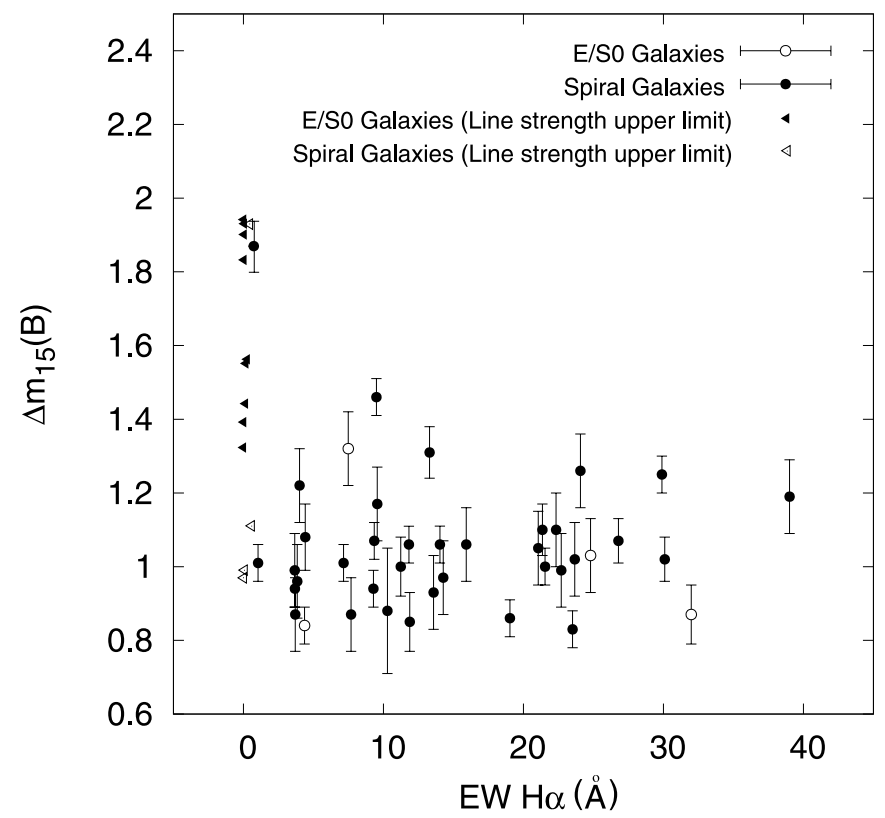

FIG. 10.- SN decline rate vs. $\mathrm{H} \alpha \mathrm{EW}$ showing the tendency for high-SFR galaxies to host only mid-range to slow-declining supernovae. The gap present in the center of the distribution may suggest two distinct populations of Type Ia SNe.

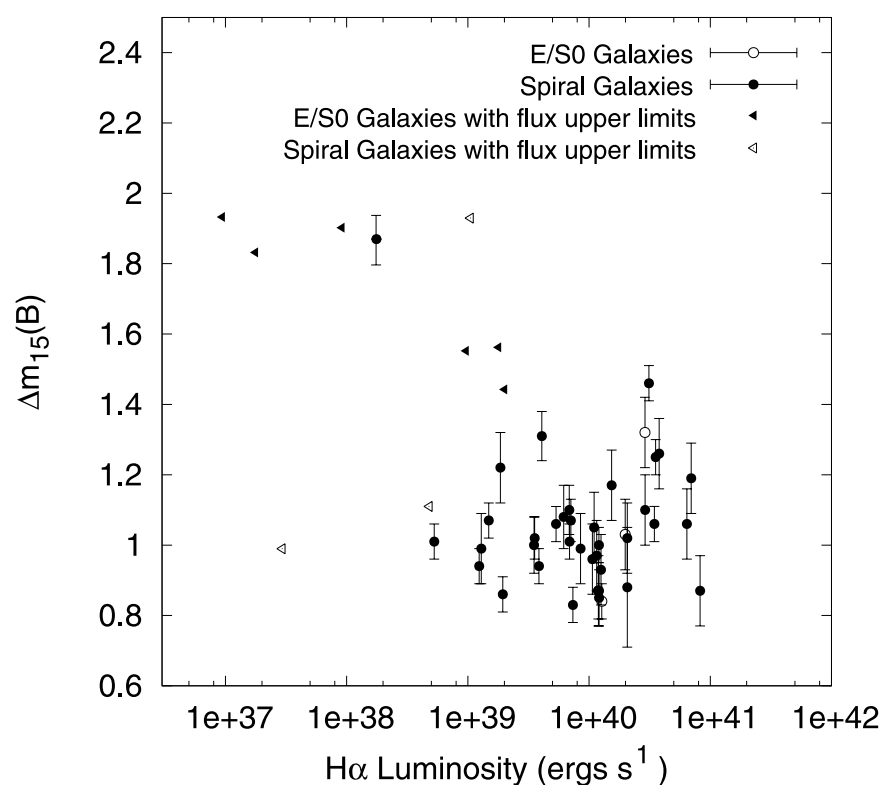

FIG. 11.- $\mathrm{H} \alpha$ luminosity vs. $\Delta m_{15}(B)$.

two phenomena through a modified Schechter function (MSF) represented by the product of these two functions governing the biases. This MSF, Figure 9, represents an approximate probability distribution governing the most likely absolute magnitudes for galaxies hosting SNe Ia. The MSF does a reasonable job in predicting the absolute magnitude distribution range for our set of SN Ia host galaxies (Fig. 8).

\subsubsection{Scalo Birthrate Parameter}

The $\mathrm{H} \alpha \mathrm{EW}$ versus $\Delta m_{15}(B)$ is given in Figure 10, while the $\mathrm{H} \alpha$ luminosity is plotted against decline rate in Figure 11. The galaxies with no discernible $\mathrm{H} \alpha$ emission are shown with their upper limits. They illustrate the propensity for the fastest declining Type Ia SNe to occur in low-emission galaxies. This result suggests that the current star formation is a galactic property at least

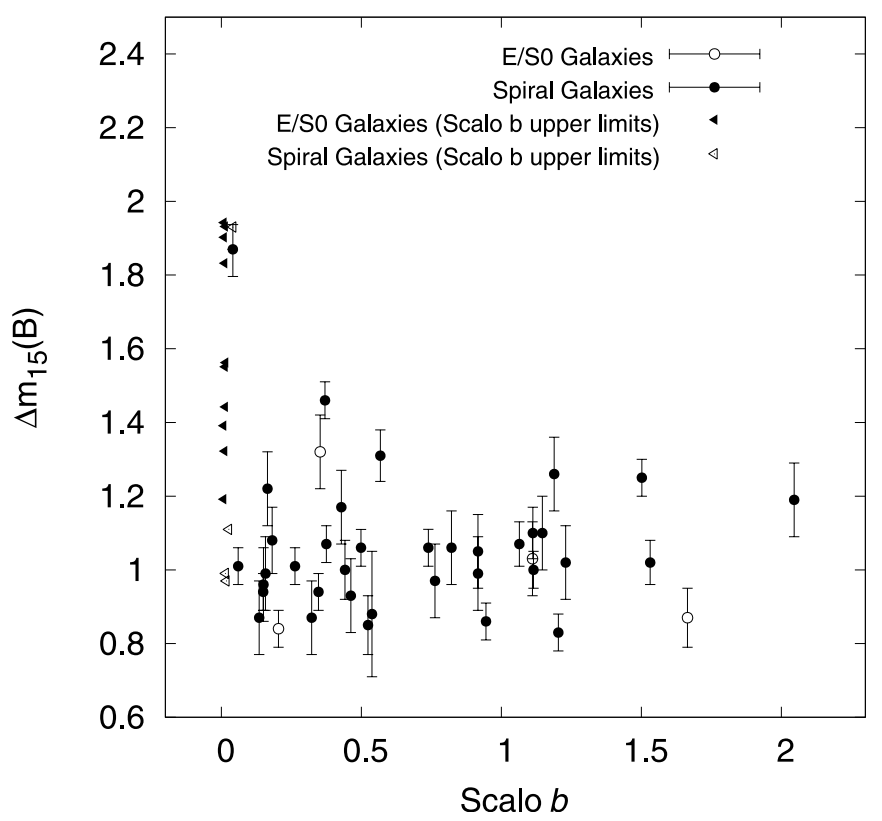

FIG. 12.-Scalo $b$ parameter (Scalo 1986) vs. SN Ia light-curve decline rate. Scalo $b$ represents the current star formation relative to average past star formation. 


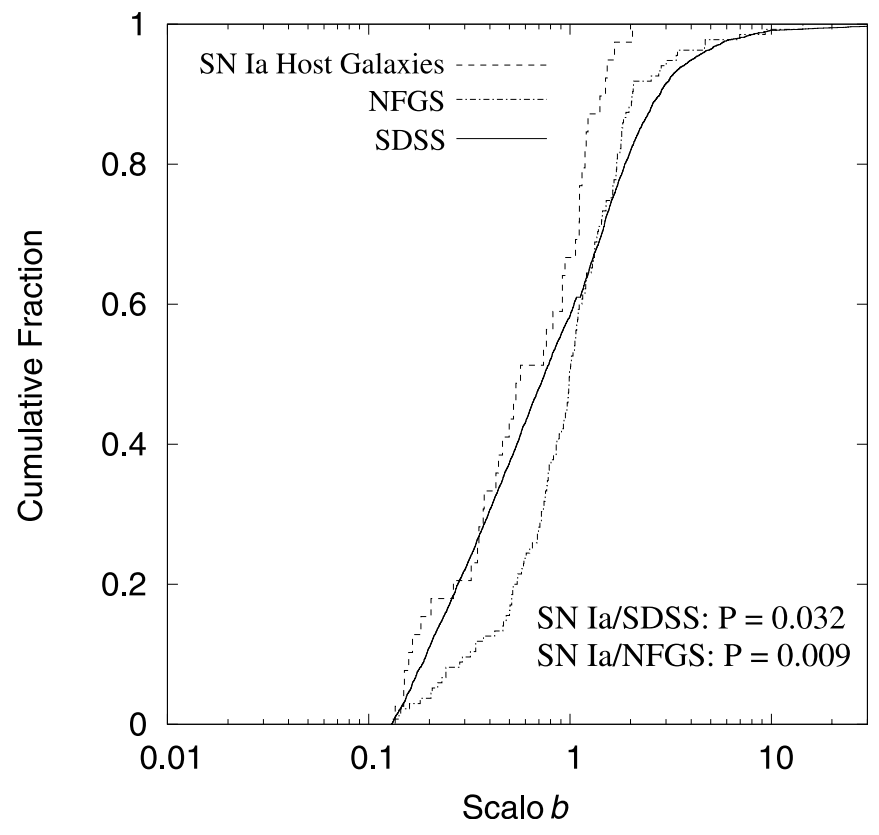

FIG. 13.-Cumulative fraction plots for our Type Ia SNe host galaxy sample, the NFGS sample of galaxies, and the SDSS galaxy sample.

partly responsible for the trend discovered by $\mathrm{H} 00$ and seen in Figure 1. Moreover, Figure 10 shows the $\mathrm{H} \alpha$ distribution to be bimodal. The presence of a gap around an equivalent width of $18 \AA$ suggests the possibility that there are two distinct populations of Type Ia supernovae. Scalo $b$ is plotted against decline rate in Figure 12. Recalling the definition of Scalo $b$, we know that current star formation relative to that of the past increases with increasing $b$. Such a gap might suggest the existence of one type of SN with a short delay time residing in high-SFR galaxies and another type having a longer delay time residing in low-SFR galaxies.

Figure 13 shows the cumulative fraction plots for the three distributions of Scalo $b$. Our goal was to compare the inherent differences between the galaxies of these three surveys; therefore, it was important to minimize the sample differences brought about by selection effects present in the design of each survey. One such selection effect apparent in the NFGS was that the quoted line strengths reported by J00 did not include specified upper limits. Such was not a reasonable expectation for the automated line-fitting procedures used by SDSS. Consequently, weak line strengths that may not have been recorded in the NFGS would have been recorded by the SDSS. This would increase the relative populations of low-SFR galaxies in the SDSS compared to the NFGS, and it would ultimately introduce uncertainty into our sample comparison. Therefore, we imposed a low-end cutoff in the Ia host galaxy sample and the SDSS sample corresponding to the lowest Scalo $b$ present in the NFGS. Although this prevented our ability to test the distributions among low-SFR galaxies, it did ensure that the three distributions could be accurately compared at high Scalo $b$ without the introduction of uncertainties due to inconsistent survey design at low Scalo $b$. The result of the K-S test reveals the probabilities of the host galaxy distribution being drawn from the same distributions as the SDSS and the NFGS sample to be $3.2 \%$ and $0.9 \%$, respectively.

Qualitatively comparing the NFGS and SNe Ia host galaxy sample leads to the following differences. The aforementioned gap in $\mathrm{H} \alpha$ and consequently in Scalo $b$, along with a relative lack of low-SFR galaxies in the NFGS, result in the SN Ia cumulative fraction plot increasing at a more rapid rate than the NFGS CFP.
The second and more intriguing difference is the lack of highSFR galaxies in the SN Ia host galaxy sample. Although similar at moderate Scalo $b$, the same disparity is seen between the SN Ia host sample and the SDSS sample at high Scalo $b$. Both the NFGS and the SDSS distribution turn over around $b=2.0$, indicating the presence of high-SFR galaxies in these respective samples. It is for this reason that the K-S test yields such low probability for the null hypothesis that the SN Ia distributions were drawn from the SDSS sample. This is also apparent in Figure 14, showing the Scalo $b$ histograms for the three distributions.

The similarities observed between the SDSS sample and the Ia host sample at moderate Scalo $b$, combined with the intentionally nonpartial nature of the NFGS, suggest that the difference between the NFGS and the SN Ia host galaxy sample at low relative SFR does not suggest a meaningful discrepancy between SN Ia hosts and the general galaxy population. In reality, the difference is most likely a consequence of the NFGS selection process. KTC94 showed that Scalo $b$ is correlated with galaxy morphological type. Consequently, we would expect a distribution of galaxies chosen to be uniform in morphological type and luminosity, such as the NFGS, to be likewise uniform in Scalo $b$.

However, the nature of the SN Ia host distribution at high Scalo $b$ is inconsistent with that observed in both the NFGS and the SDSS galaxy samples. Galaxies with current star formation higher than approximately 2 times the average past SFR seem to be selected out of the SN Ia host galaxy sample. A Monte Carlo test gives the probability of selecting 39 Scalo $b$ values at random from the SDSS sample and fortuitously obtaining all values below 2.05 to be $\sim 0.05 \%$. Such a result implies a high significance for this cutoff.

A property of Type Ia supernovae that has the potential to explain this rejection is the SN delay time, or alternately the time between progenitor formation and the supernova event. We investigated the implications of this cutoff given the following assumptions: (1) The high-SFR galaxies in the SN Ia sample have SFHs that are described by an exponential decline in SFR followed by a recent burst. We assume that the progenitor for each SN Ia hosted by a high-SFR galaxy was thus formed during the current burst. (2) There is a direct correlation between the number of SDSS galaxies observed in a particular Scalo $b$ range (Fig. 14) and the duration over which the average galaxy spends producing stars at that SFR ( $\left.t_{\text {burst }}\right)$. And (3) The ratio of the duration of a burst, with its peak at Scalo $b=b^{\prime}$, to the total lifetime of the galaxy ( $\sim 14 \mathrm{Gyr}$ ) can be found through evaluation of the following:

$$
\text { Ratio }=\frac{\int_{b^{\prime}}^{\infty} t_{\text {burst }}(b) d b}{\int_{0}^{\infty} t_{\text {burst }}(b) d b} .
$$

Given these three assumptions it is possible to place an approximate lower limit on the $\mathrm{SN}$ Ia delay time, $\tau_{\text {min. }}$. Our observed cutoff might suggest that galaxies with current SFRs higher than $b \sim 2.0$ are in the midst of a star formation burst that is too short to have created an SN Ia progenitor, while allowing ample time for it to evolve and explode. By approximating an average galactic lifetime of $14 \mathrm{Gyr}$, an evaluation of equation (3) for $b^{\prime}=2.0$ results in a $\tau_{\min } \sim 2.0$ Gyr. Recent work conducted by Gal-Yam \& Maoz (2004) attempted to constrain $\tau_{\min }$ by comparing the theory and observation of the SN Ia redshift distribution. Beginning with theoretical functions governing the SFH and SN Ia delay time, they calculated the expected redshift distribution of SNe Ia and compared this to the observed distribution of supernovae discovered by the Supernova Cosmology Project. The SFH function and SN Ia delay function are degenerate and thus could not be simultaneously constrained. 

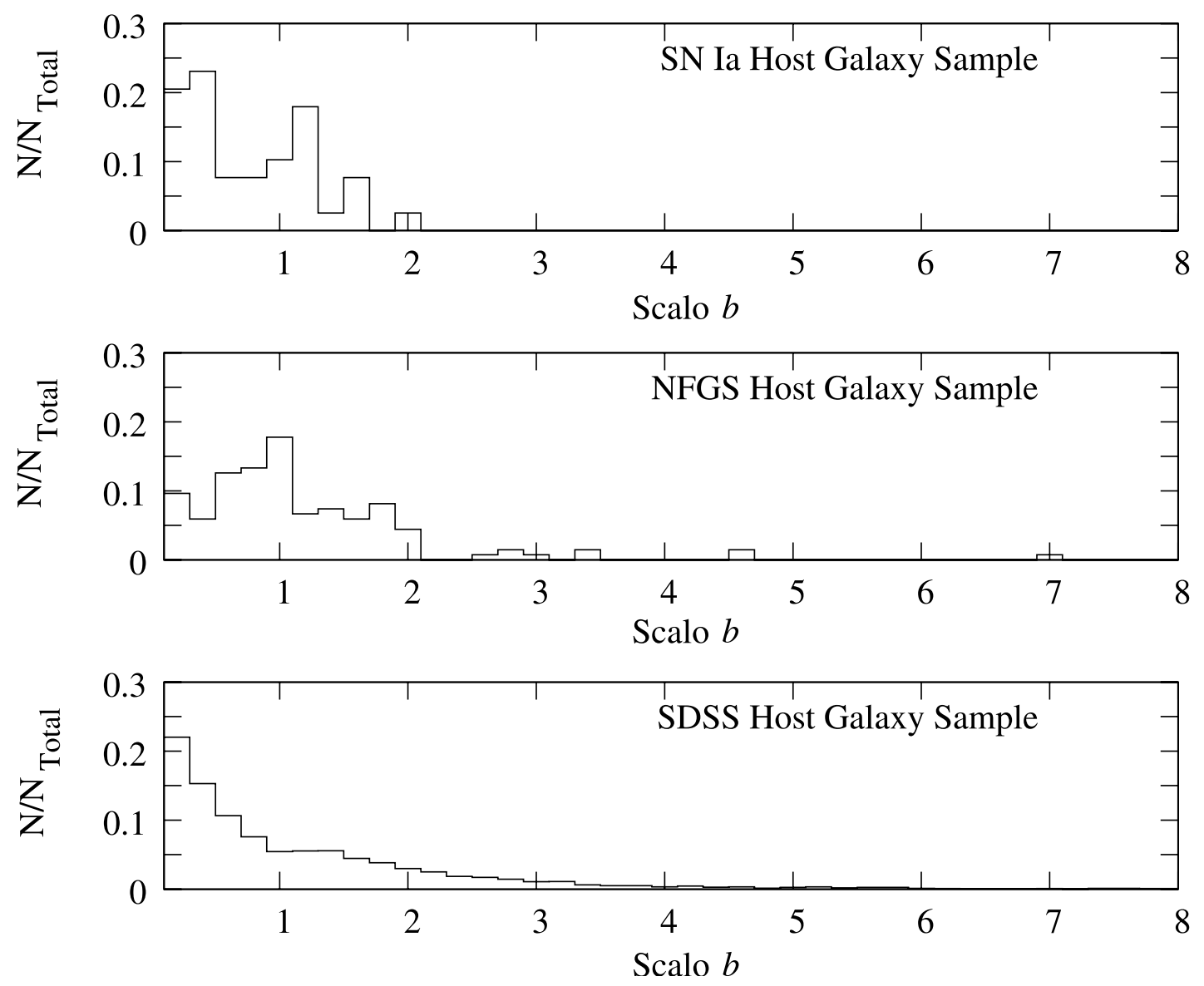

FIG. 14. - Scalo $b$ distribution in the SN Ia host sample, the NFGS sample, and the SDSS sample. Note the cutoff in the SNe Ia host galaxy sample at $b \sim 2$.

A SFH function had to be assumed in order to constrain the SN Ia delay function and vice versa. Assuming the SFH function predicted by Madau et al. (1998), the results predict $\tau_{\min }=1.7 h^{-1}$ Gyr ( $\sim 1.2$ Gyr) at the $95 \%$ confidence level. However, they go on to show that a longer characteristic delay time lower limit, $\tau_{\min } \geq 3 h^{-1}$ Gyr $(2.1 \mathrm{Gyr})$, is allowed if the SFH model of Lanzetta et al. (2002) is assumed.

\subsection{Hubble Residual}

Assuming a positive correlation between galactic and progenitor metallicity, our results indicate that it is unlikely that variations in progenitor metallicity can entirely account for the large brightness variations observed in SNe Ia at peak luminosity. Although not a primary contributor, metallicity may contribute to more subtle variations at the level of the SN Ia intrinsic scatter. This can be tested by plotting the SN Hubble residuals, which are expected to have variations on the order of $0.18 \mathrm{mag}$ (Jha 2002), versus galactic metallicity (Fig, 15a). The plot appears to show a slight trend for higher metallicity galaxies to produce $\mathrm{SNe}$ Ia with negative Hubble diagram residual. A Monte Carlo simulation reveals this correlation amplitude to be $90 \%$. The test was conducted as follows. We generated 16 Gaussian-distributed random numbers using a standard deviation of 0.18 and assigned to each one a metallicity measurement from our data. We then plotted the metallicity versus this theoretical residual and calculated the slope of the best-fit line. Repeated trials allowed us to determined the likelihood of obtaining a best-fit slope to the data greater than or equal to the absolute value of the slope observed in the best fit to Figure $15 a$. This is a less than $2 \sigma$ detection and should be treated with caution.
However, it could suggest that metallicity is a secondary parameter affecting the brightness of $\mathrm{SNe}$ Ia at the $10 \%$ level. Nevertheless, continued study is needed to bring about a more conclusive understanding. Figures $15 a-15 c$ show similar plots for absolute magnitude, Scalo $b$, and the $\log$ of $\mathrm{H} \alpha$ luminosity. We found insignificant correlations for these three parameters, with Monte Carlo simulations placing the linear fits near the $75 \%, 75 \%$, and $50 \%$ confidence levels, respectively.

\subsection{Summary}

We have analyzed the globally integrated spectra for a sample of Type Ia supernova host galaxies in order to investigate the possible systematic effects that the host galaxy environment has on the properties of Type Ia supernovae. We looked for direct correlations between the decline rates of Type Ia supernovae and host galaxy metallicity, absolute $B$ filter magnitude, and SFR. We further looked for correlations between these galactic parameters and the resident supernova's Hubble best-fit residual. Finally, we investigated the systematic differences between SN Ia host galaxies and the general galactic population through a series of Kolmogorov-Smirnov tests. The main results are as follows:

1. We find no correlation between spiral galaxy metallicity and SN Ia decline rate. We find significant decline rate variability at fixed host galaxy metallicity, implying a small impact of metallicity on the peak luminosity of Type Ia supernovae. We find that SNe at smaller PGDs have a higher average luminosity than those residing further from their galaxy's nucleus. As metallicity is predicted to decrease as a function of PGD in spiral galaxies, this result stands in contrast to the predictions made by combining 

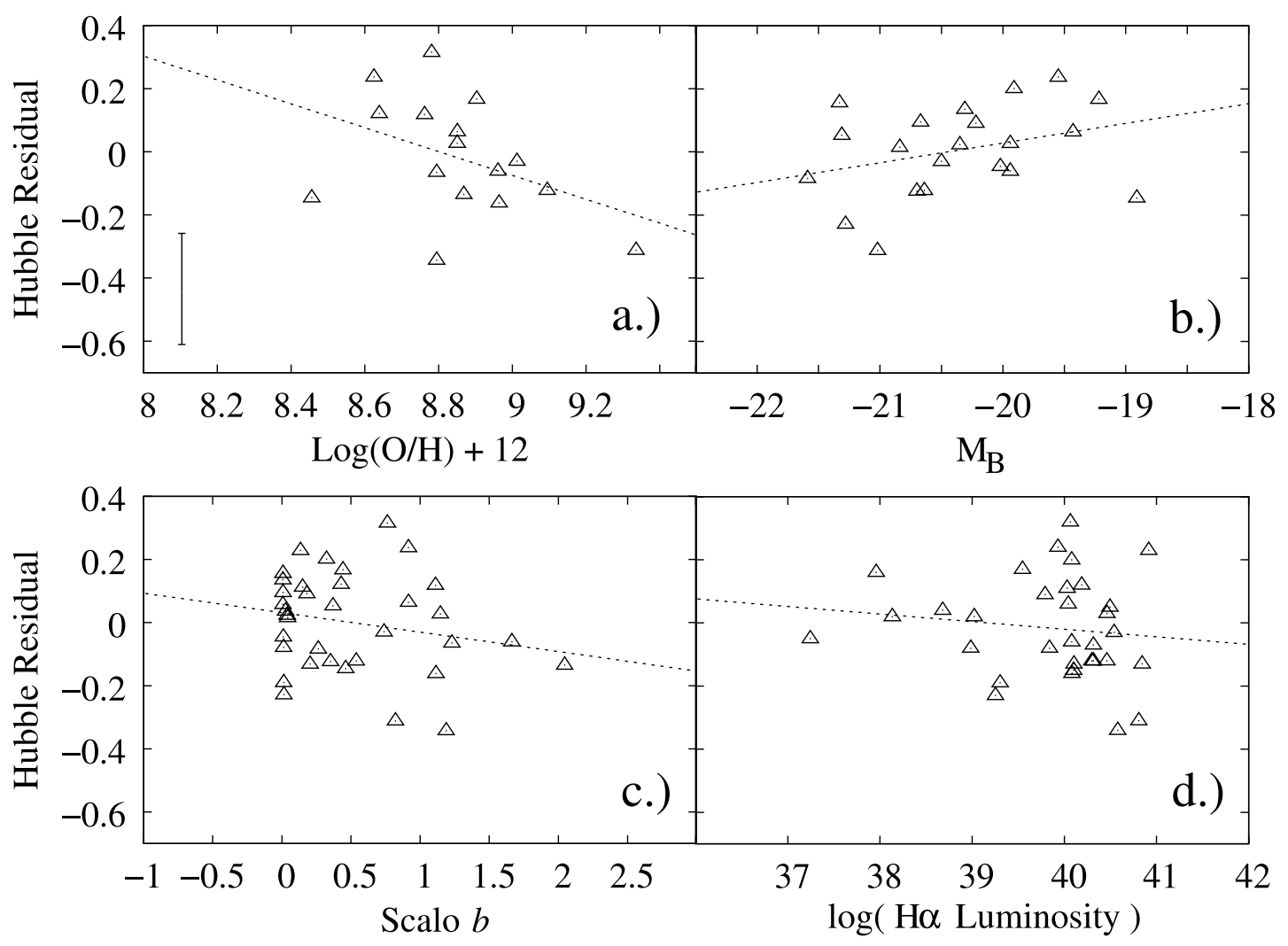

FIG. 15.-Systematic errors present in the SN Hubble residuals. The figure shows the relationship between Hubble residual and host galaxy metallicity ( $a$ ), host

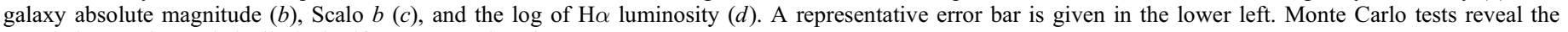
correlations to be statistically insignificant to varying degrees.

the results of TBT03, Höflich et al. (2002), and Garnavich et al. (2004). Furthermore, a K-S test shows the SN Ia host galaxy metallicity distribution to be statistically similar to the NFGS and particularly to the SDSS metallicity distributions. Our results also indicate that the progenitor age can have a significant impact on the variations in Type Ia decline rates. Assuming that the global galactic metallicity is approximately correlated with the progenitor metallicity, this implies that it is the age, and not the metallicity, of the progenitor that is the greater contributor to the inhomogeneities in Type Ia supernovae explosions.

2. The gradual trend found by H0O between host galaxy absolute magnitude and supernova decline rate is not seen in our galaxy sample. Nevertheless, similar to the results of H00, we do find a lack of faint $\mathrm{SNe}$ in the low-luminosity galaxy regime. This is most likely a combination of the expected lack of $\mathrm{SNe}$ in small galaxies and the behavior observed in Figure 1, which shows the tendency for low-luminosity SNe Ia to be hosted almost exclusively by early-type galaxies. Since ellipticals are on average brighter than other galaxies on the Hubble sequence, we would expect low-luminosity SNe Ia to confine themselves to brighter host galaxies. The average absolute $B$ magnitude of our host galaxies are found to be systematically brighter than the galaxies of the NFGS. The phenomenon can be attributed to the combined selection effect due to the increased population of stars in high-luminosity galaxies and the nonuniformity of the luminosity function of galaxies.

3. We do not see evidence for a dependency of supernova decline rate on Scalo $b$ in host galaxies with active star formation. However, we do see a discontinuity in the relationship between SFR and SFH to the extent that all of the fast-declining $\mathrm{SNe}$ Ia studied have been hosted by galaxies with low to non- existent star formation. Moreover, the distribution of Scalo $b$ for the host galaxy sample shows bimodal behavior, suggesting the possibility of two distinct populations of Type Ia supernovae possessing different delay times. The host galaxy Scalo $b$ distribution also shows an unexpected cutoff at $b \sim 2$, which might be indicative of a finite lower limit on the delay time of Type Ia supernovae. We approximate this lower limit to be $2.0 \mathrm{Gyr}$, slightly higher than the best value of 1.2 Gyr obtained by Gal-Yam \& Maoz (2004). Future refinements to the SFH models should help to finally pin down the true characteristic delay time.

4. Our tests to determine the effects of host galaxy environment on the SN Hubble residual all proved to be inconclusive to varying degrees. However, a $90 \%$ significance to a trend tying metallicity to Hubble residual, though requiring more study to prove or disprove, could suggest that metallicity, while not likely responsible for the diversity of SNe Ia brightnesses on the order of $1 \mathrm{mag}$, could be responsible for more subtle brightness variation seen at the $0.1 \mathrm{mag}$ level. A continuation of this work currently underway is attempting to increase the host galaxy population used in this study which will enable us to improve the likelihood of detecting statistically significant results.

We would like to thank Lisa J. Kewley for supplying an IDL script used to perform host galaxy extinction corrections and metallicity determination. Partial funding for this project was supplied by the Notre Dame Center for Applied Mathematics. This research has made use of the NASA/IPAC Extragalactic Database (NED), which is operated by the Jet Propulsion Laboratory, California Institute of Technology, under contract with the National Aeronautics and Space Administration. 


\section{REFERENCES}

Andrievsky, S. M., Luck, R. E., Martin, P., \& Lépine, J. R. D. 2004, A\&A, 413, 159

Caldwell, C. N., \& Oemler, A. 1981, AJ, 86, 1424

Dominguez, I., Chieffi, A., \& Straniero, O. 1999, ApJ, 524, 226

Fabricant, D., Cheimets, P., Caldwell, N., \& Geary, J. 1998, PASP, 110, 79

Filippenko, A. V. 1982, PASP, 94, 715

Freedman, W. L., et al. 2001, ApJ, 553, 47

Gal-Yam, A., \& Maoz, D. 2004, MNRAS, 347, 942

Garnavich, P. M., et al. 1998, ApJ, 509, 74 .2004, ApJ, 613, 1120

Geller, M., et al. 1997, AJ, 114, 2205

Gibson, B. K., et al. 2000, ApJ, 529, 723

Hamuy, M., Phillips, M. M., Suntzeff, N. B., Schommer, R. A., Maza, J., \& Aviles, R. 1996a, AJ, 112, 2391 . 1996b, AJ, 112, 2398

Hamuy, M., et al. 2000, AJ, 120, 1479 (H00)

Henry, R. B. C., \& Worthey, G. 1999, PASP, 111, 919

Höflich, P., Gerardy, C. L., Fesen, R. A., \& Sakai, S. 2002, ApJ, 568, 791

Höflich, P., Nomoto, K., Umeda, H., \& Wheeler, J. C. 2000, ApJ, 528, 590 (HNUW00)

Höflich, P., Wheeler, J. C., \& Thielemann, F. K. 1998, ApJ, 495, 617

Iben, I., Jr., \& Tutukov, A. V. 1984, ApJS, 54, 335

Jansen, R. A., Fabricant, D., Franx, M., \& Caldwell, N. 2000, ApJS, 126, 331 (J00)

Jha, S. 2002, Ph.D. thesis, Harvard Univ.

Jha, S., et al. 1999, ApJS, 125, 73

Kennicutt, R. C., Tamblyn, P., \& Congdon, C. W. 1994, ApJ, 435, 22 (KTC94)

Kewley, L. J., \& Dopita, M. A. 2002, ApJS, 142, 35

Khokhlov, A. M. 1991, A\&A, 245, 114

Krisciunas, K., Phillips, M. M., \& Suntzeff, N. B. 2004, ApJ, 602, L81

Kroupa, P., Tout, C. A., \& Gilmore, G. 1993, MNRAS, 262, 545

Lanzetta, K. M., Yahata, N., Pascarelle, S., Chen, H., \& Fernãndez-Soto, A. 2002, ApJ, 570, 492

Madau, P., et al. 1998, ApJ, 498, 106

Mannucci, F., et al. 2005, A\&A, 433, 807

Massey, P., Strobel, K., Barnes, J. V., \& Anderson, E. 1988, ApJ, 328, 315

McMillan, R. J., \& Ciardullo, R. 1996, ApJ, 473, 707

Miller, M. S., \& Mathews, W. G. 1972, ApJ, 172, 593

Nomoto, K. 1982, ApJ, 253, 798

Oemler, A., \& Tinsley, B. M. 1979, AJ, 84, 985

Perlmutter, S., et al. 1997, ApJ, 483, 565 . 1999, ApJ, 517, 565

Phillips, M. M. 1993, ApJ, 413, L105

Phillips, M. M., Lira, P., Suntzeff, N. B., Schommer, R. A., Hamuy, M., \& Maza, J. 1999, AJ, 118, 1766

Qian, Y. Z., \& Wasserburg, G. J. 2001, ApJ, 549, 337

Reiss, A. G., Press, W. H., \& Kirshner, R. P. 1996, ApJ, 473, 88

Riess, A. G., et al. 1998, AJ, 116, 1009 1999, AJ, 117, 707

Scalo, J. M. 1986, Fundam. Cosmic Phys., 11, 1

Schechter, P. 1976, ApJ, 203, 297

Schmidt, B. P., et al. 1998, ApJ, 507, 46

Strolger, L.-G., et al. 2004, ApJ, 613, 200

Timmes, F. X., Brown, E. F., \& Truran, J. W. 2003, ApJ, 590, L83 (ТВT03)

Umeda, H., Nomoto, K., Kobayashi, C., Hachisu, I., \& Kato, M. 1999, ApJ, 522, L43

Wang, Y., \& Garnavich, P. M. 2001, ApJ, 552, 445

Webbink, R. F. 1984, ApJ, 277, 178

Whelan, J., \& Iben, I. Jr. 1973, ApJ, 186, 1007

Woosley, S. E., \& Weaver, T. A. 1994, in Supernovae, ed. S. A. Bludman, R. Mochkovitch, \& J. Zinn-Justin (Amsterdam: Elsevier), 423

Worthey, G. 1994, ApJS, 95, 107

Yamaoka, H. Nomoto, K., Shigeyama, T., \& Thielemann, F. 1992, ApJ, 393, L55 